\title{
Parameterization of historical earthquakes in Switzerland
}

\section{Journal Article}

Author(s):

Álvarez-Rubio, Sonia; Kästli, Philipp (D); Fäh, Donat; Sellami, Souad; Giardini, Domenico

Publication date:

2012-01

Permanent link:

https://doi.org/10.3929/ethz-b-000042011

Rights / license:

In Copyright - Non-Commercial Use Permitted

Originally published in:

Journal of Seismology 16(1), https://doi.org/10.1007/s10950-011-9245-8 


\title{
Parameterization of historical earthquakes in Switzerland
}

\author{
Sonia Álvarez-Rubio • Philipp Kästli • Donat Fäh • \\ Souad Sellami • Domenico Giardini
}

Received: 6 February 2011 / Accepted: 13 July 2011 /Published online: 5 October 2011

(C) Springer Science+Business Media B.V. 2011

\begin{abstract}
Macroseismic earthquake parameters of historical events have been reassessed in the framework of the update of the Earthquake Catalogue of Switzerland ECOS-09. The Bakun and Wentworth method (Bakun and Wentworth 1997) has been used to assess location, magnitude, and, when possible, focal depth. We apply a two-step procedure. Intensity attenuation is assessed first by fitting a model with a logarithmic and a linear term, using a set of 111 earthquakes. The magnitude range is 3 and 5.8. Then, intensity to magnitude relation is developed. A subset of the 111 events, all having an instrumental moment magnitude, was used to perform this intensity to magnitude calibration. Five final calibration strategies were developed based on
\end{abstract}

\footnotetext{
S. Álvarez-Rubio $(\square) \cdot$ P. Kästli • D. Fäh · S. Sellami •

D. Giardini

Swiss Seismological Service, ETH Zurich,

Sonneggstrasse 5,

CH-8092 Zürich, Switzerland

e-mail: sonia.alvarez@sed.ethz.ch

Sonia. Álvarez-Rubio

e-mail: sonia.alvarez.rubio@gmail.com

P. Kästli

e-mail: kaestli@sed.ethz.ch

D. Fäh

e-mail: donat.faeh@sed.ethz.ch

S. Sellami

e-mail: souad.sellami@sed.ethz.ch

D. Giardini

e-mail: domenico.giardini@sed.ethz.ch
}

different intensity calibration datasets, regionalized or non-regionalized models, and fixed or variable source depth. The final assessment of the macroseismic earthquake parameters is based on an expert judgment procedure, using the results derived from all five strategies, and taking into consideration the historical knowledge available for the particular earthquake. A bootstrap procedure has been applied to assess the uncertainty of parameters. Indicative lower and upper bounds of uncertainty are derived from distributions of location and magnitude for a number of events, obtained through bootstrap sampling of the intensity field and of the single intensity values. The final uncertainties are given in terms of parameter uncertainty classes already used in previous versions of the earthquake catalogue of Switzerland.

Keywords Macroseismic intensity $\cdot$ Historical earthquakes · Calibration · Intensity attenuation . Switzerland

\section{Introduction}

In areas of low to moderate seismicity, such as Switzerland, the majority of the large earthquakes belong to the pre-instrumental period. Only macroseismic information is available that is obtained either through questionnaires, newspaper articles, or historical documents. Macroseismic intensity data points are a semi-empirical measure for the earthquake 
effects and indirectly for earthquake size. Good quality macroseismic fields with a sufficiently large number of intensity data points are a prerequisite for a reliable assessment of earthquake source parameters.

The core of the earthquake catalogue of Switzerland ECOS-09 is the Macroseismic Earthquake Catalogue of Switzerland (MECOS). It includes a database of historical and macroseismic information built upon the collection and analysis of historical data. It merges all available macroseismic inputs for significant events within Switzerland. After the first publication of the entire macroseismic dataset with ECOS-02 in 2002 (Fäh et al. 2003; Swiss Seismological Service 2002), a complete revision has been undertaken in order to create a refined version of the catalogue (Fäh et al. 2011; Gisler and Fäh 2011; Schwarz-Zanetti and Fäh 2011). Between 2003 and 2009, numerous additional information was collected and stored in the database, covering both recent earthquakes (2003 through to 2009) as well as historical ones (i.e., earthquakes before 1975). Since the database is for internal use only, it was the ambition to publish as much of the knowledge as possible, in order to provide it to interested parties. In particular, the historical information and macroseismic data is now outlined in two volumes, covering the time period between 1000 and 1878 . The first part (Schwarz-Zanetti and Fäh 2011) summarizes the knowledge of events roughly between 1000 and 1680, in other words, from the first traces in the Middle Ages to the early Enlightenment. The second part (Gisler and Fäh 2011) summarizes the events between 1680 and 1878 , covering the enlightenment era and the nineteenth century and ending with the year when the Swiss Seismological Commission was established. For the period after 1878, all known events are summarized in the annual bulletins of the Swiss Seismological Service (Schweizerische Erdbebenkommission/Schweizerischer Erdbebendienst, 1881-1962; 1972-1974).

A homogeneous quality level is kept in the two volumes, for the macroseismic database and catalogue ECOS-09, using the European macroseismic scale (EMS-98). This allowed establishing a macroseismic field for each event. For many events, a most probable intensity was assigned to each locality and a range of intensity by giving both minimum and maximum possible values. This range illuminates information gaps and uncertainties regarding historical interpretation. As outlined in this paper, this uncertainty in intensity data is used to assess the uncertainties of the derived earthquake source parameters. In addition, intensity data points were imported into the database from available compilations as described in the ECOS-02 reports (Swiss Seismological Service 2002). Foreign intensity data points were used when calibrating border events. Within and outside Switzerland, preference is given to intensity data points if analyzed by the team of the Swiss Seismological Service.

Many different intensity-based approaches have been developed in the past to estimate the earthquake source parameters needed for seismic hazard assessment (e.g., Musson et al. 2008). The quality of the derived parameters is, on the one hand, depending on the quantity, quality, and distribution of the intensity data points in the macroseismic field, and on the other hand, on the method applied for the estimation of such parameter.

The Swiss Seismological Service (SED) performed preparatory research on the parameterization of historical earthquakes within the framework of the NERIES project in work package NA4 distributed archive of historical earthquake data (Stucchi 2010). Part of this work was a calibration initiative for improving the determination of earthquake parameters from macroseismic data (Gómez Capera et al. 2009). The procedures included various published and unpublished methods, using repeatable methods and homogeneous input data.

The techniques selected for the calibration were the method proposed by Bakun and Wentworth (hereafter BW, Bakun and Wentworth 1997; Hinzen and Oemisch 2001; Fäh et al. 2003; Bakun et al. 2003; Bakun and Scotti 2006; Stromeyer and Grünthal 2009), the Boxer method (Gasperini et al 1999; Gasperini 2004), and the MEEP method (Macroseismic Estimation of Earthquake Parameters, Musson et al. 2008). They have been applied in five European areas: Aegean, Iberia, Italy, Great Britain, and Switzerland.

From the experience during this project, we selected the BW method for the final calibration of historical parameterization, following therefore the same strategy as for ECOS-02 (Fäh et al. 2003). The Boxer method was applied to validate results from BW.

The key issue in the application of BW method, as well as in all other methods, is the calibration procedure. Macroseismic intensity is an ordinal rather than quantitative measure that may include issues related to the national practice to assign intensity, 
information on building quality, and population density. Although national databases are rather homogeneous, the intensity values might therefore change from region to region, and there might be differences specifically in border areas (Stucchi 2010). Additionally, different macroseismic scales used through time or, in particular, regions might introduce inconsistencies. These aspects of the nature of seismic intensity and related source parameters are discussed in detail in Pasolini et al. (2008a). It is evident from the nature of intensity data that no metric exists in the field of intensity values. This has to be recalled when deriving attenuation relationships.

We went through a selective calibration process in terms of the selection of the calibration dataset, the intensity attenuation formulation, and the intensity to magnitude relation. Besides assessing macroseismic location and magnitude, we have addressed the estimation of depth as a variable of the intensity attenuation model and estimated as well the uncertainty of source parameters.

A strategy for the final assessment of macroseismic earthquake parameters of historical events was developed based on five calibration procedures. The magnitude estimates obtained with the five procedures for the same event are variable in the sense that firstly there is considerable scatter, and secondly, there is no single calibration strategy that performs the best for all events. Location and magnitude have therefore been assessed using expert judgment based on the distribution of locations and magnitudes derived from the different strategies, as well as on the seismotectonic setting and our knowledge from historical sources. The calibration work is outlined in the following chapters. Further information can be found in the catalogue section of the website http:// www.seismo.ethz.ch (Fäh et al. 2011).

\section{Calibration procedure}

Parameterization of historical earthquakes (epicenter location, magnitude, and depth if possible) is performed with the BW method. The procedure is based on a two-stage calibration that decouples the estimation of the distance dependency of the intensity field, from the estimation of macroseismic magnitude. We first tested several attenuation models based on Köveslighety (1906), Blake (1941), and Gómez
Capera (2006) that comprise different variables and functional forms:

$I_{\mathrm{obs}}-I_{\mathrm{sc}}=f(d, h)$

where $I_{\mathrm{obs}}$ refers to the observed intensities, $I_{\mathrm{sc}}$ is the event-individual scaling intensity, which is derived in an iterative regression process together with a set of the coefficients of the function $f$. The term $d$ stands for epicentral distance $(D)$ or hypocentral distance $(R)$, and $h$ refers to focal depth. $I_{\mathrm{sc}}$ is the link or scaling between the attenuation model to be calibrated and the magnitude of the event. Different formulations (1) were assessed using selected calibration datasets from all macroseismic data available. Three different subsets have been then used for calibration.

The first calibration dataset consists of events with known location and sufficiently dense intensity fields. They are mainly the instrumentally located events of the twentieth century with local magnitude larger than 3.2. However, older events from the nineteenth and twentieth centuries are added to the dataset if the macroseismic field contains at least 10 intensity data points (IDPs) with intensity greater than or equal to 3 . This calibration dataset 1 is the largest.

This dataset was consecutively reduced using the following restriction criteria: events with a small number or heterogeneous distribution of IDPs, events with a restricted number of intensity classes, and events with intensity fields with anomalously high intensity levels far from the epicenter are removed from the calibration dataset. The reduced dataset is called dataset 2. Finally, all events that did not fit the attenuation relations well were removed from dataset 2 , and this defines the smallest set dataset 3 . This reduction allows an improvement of the fit of the attenuation curve to the intensity data points according to Eq.1. For these two last datasets, a regionalization in alpine and foreland events has been also established (Fig. 1).

Figure 2 is a representation of the number of intensity points of dataset 1 in the different magnitude ranges. Table 1 shows the different intensity attenuation models that have been calibrated and tested. For those formulations where a term depends on depth $h$, we have addressed the calibration of the attenuation coefficients $a, b$, following the strategies:

(a) $h$ is fixed at $10 \mathrm{~km}$ (fixed-depth strategy) 
Fig. 1 Geographical distribution of the events in the calibration datasets. a All events shown correspond to dataset 1; events in dark blue are the sub-selection dataset 2. b Green: events with assigned instrumental magnitude, $M_{w}$ (bestmag) used for magnitude calibration; gray: all events included in the final assessment of macroseismic parameters in ECOS-09
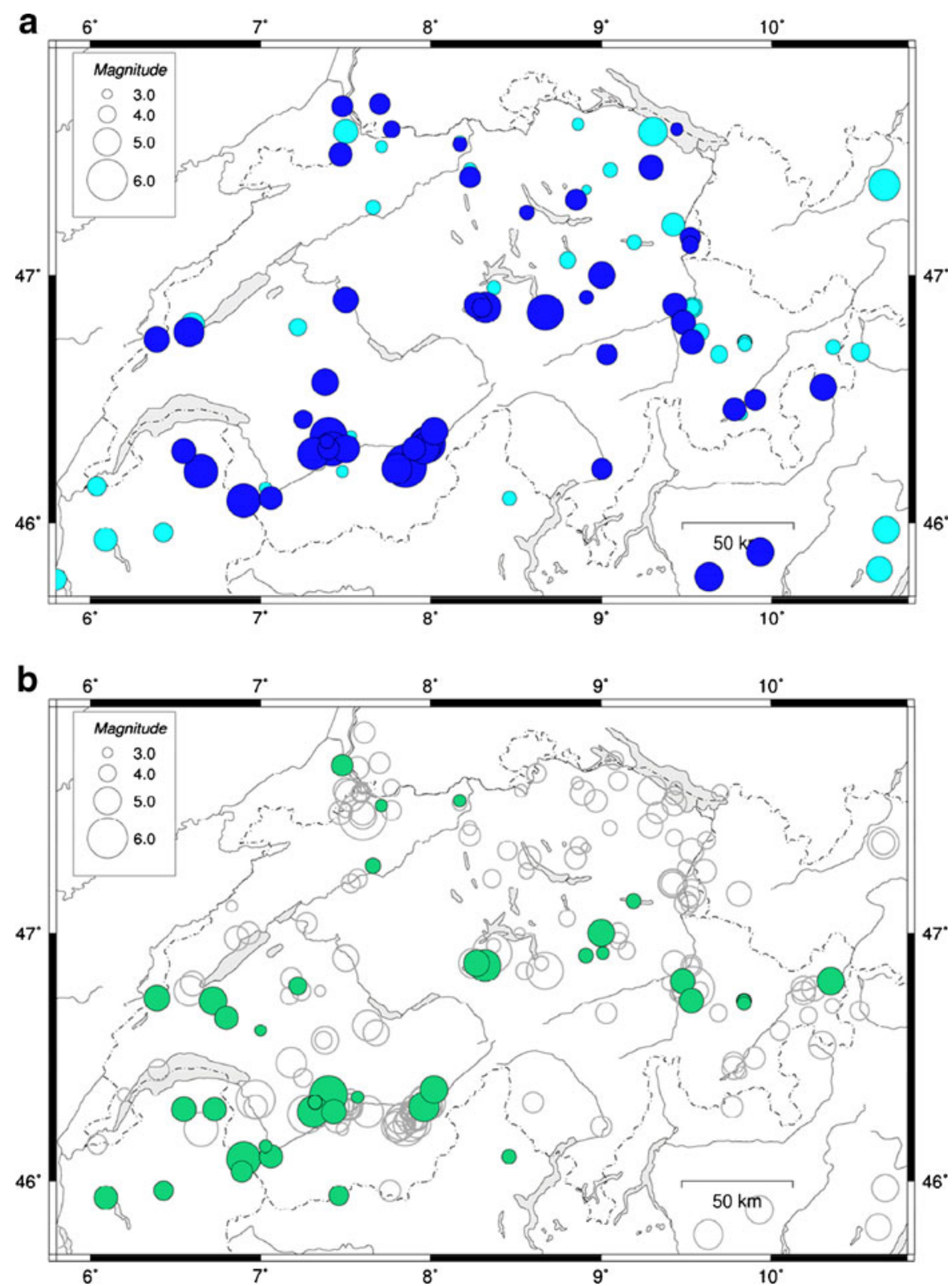

(b) $h$ is allowed to vary from 3 to $25 \mathrm{~km}$ (variable-depth strategy)

(c) $h$ is fixed for those calibration events where depth is known from instrumental determination, while for the other events depth is used as a fitting parameter (mixed strategy) still using depth constraints.

We analyzed results derived from binned (distance and intensity) and non-binned intensity representations (see Bakun and Scotti 2006). Finally, the intensity representation used is "no-binning" due to its consis- tency with the application of the BW technique for the assessment of the macroseismic parameters. We tested several weighting schemes to avoid the influence of the incompleteness within certain intensity classes and to account for the increase in area with distance. Finally, we applied a quadratic weight, decaying within $200 \mathrm{~km}$ $w=(200-d / 200)^{2}$, similar to the cosine-based weighting scheme used in Bakun's search algorithm to calibrate the events in ECOS-02 (Fäh et al. 2003). We addressed strategies based on cutoff intensities that led to a selection of a subset of the entire intensity 
Fig. 2 Magnitude distribution of the intensity data points in calibration dataset 1

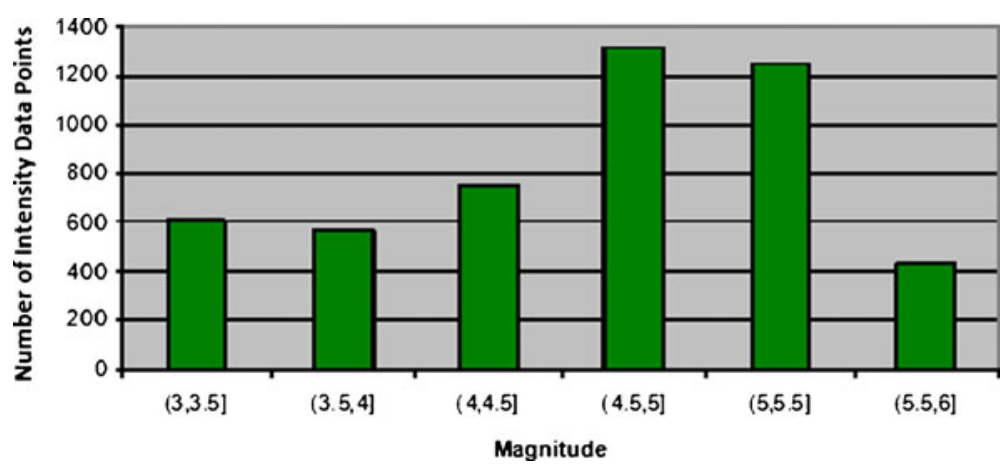

field, used then to fit the attenuation model. The strategies include:

(a) Using all IDPs with intensities $\geq 3$ (allint strategy);

(b) Using only the three highest intensity levels (top3 strategy) for each event;

(c) Using only the IDPs with intensities between 4 and 6 (int4-6 strategy). Even if the performance of this strategy was good (as it is little affected by heterogeneities in the intensity scale itself), it was finally not implemented due to the fact that many intensity fields of historical events in earlier times are not complete for the intensity 4-6 range.

Figure 3 summarizes all aspects of the calibration of the intensity attenuation models that were tested using the functional forms given in Table 1. As it is shown, each calibration strategy can be defined in terms of the attenuation model, the calibration dataset, the regionalization, the intensity field representation, and the depth strategy.

In a second step, a relation between magnitude $(M)$ and event-individual scaling intensity at a standard distance was developed for the attenuation models:

$M=\alpha I_{30}+\beta$

Table 1 Intensity attenuation models tested in ECOS-09

\begin{tabular}{ll}
\hline Functional form & Intensity attenuation models \\
\hline Logarithmic and linear & $I-I_{\mathrm{sc}}=a \operatorname{Ln}\left(\frac{d^{\mathrm{a}}}{h}\right)+b\left(d^{\mathrm{a}}-h\right)$ \\
Logarithmic & $I-I_{\mathrm{sc}}=a \operatorname{Ln}(R)+b$ \\
Linear & $I-I_{\mathrm{sc}}=a D+b$ \\
Cubic & $I-I_{\mathrm{sc}}=a \sqrt[3]{R}+b$
\end{tabular}

$D$ epicentral distance, $R$ hypocentral distance, $a, b$ calibrated coefficients

${ }^{\text {a }}$ This functional form has been tested for both distances $D$ and $R$
The standard distance is chosen to be $30 \mathrm{~km}$ hypocentral distance $\left(I_{30}\right)$. The influence of the focal depth on the estimation of the magnitude is then significantly reduced because events in Switzerland occur at depths smaller than $30 \mathrm{~km}$.

From the above-mentioned calibration datasets, we defined a subset of events, which all have instrumentally derived moment magnitude, $M_{\mathrm{w}}$, hereafter known as $M_{w}$ (bestmag). This calibration dataset has been used to derive $\alpha$ and $\beta$ in the standardized intensity to macroseismic magnitude calibration (Eq.2).

The magnitude calibration in Eq.2 performed with events with $M_{w}$ (bestmag) was carried out for each strategy that was finally selected; see Fig. 3. Moreover, this regression was performed following three schemes for weighting a single-event contribution in the assessment of the $I_{s c}$-to-magnitude relation: (a) equal weighting of all events, (b) weighting of each event by the number of intensity data points used for in the inversion for $I_{\mathrm{sc}}$, and (c) additional weighting by the quality of each intensity data point (ordinal scale for weight 1 [very poor information] to weight 5 [high quality supported by a large number of observational data at the site of the intensity data point]).

In the next chapter, the performance of the different models and strategies is discussed, and the justification for the selection of five calibration strategies is outlined.

\section{Results of the calibration: ECOS-09 calibration strategy}

The calibration procedure has been a complex process in terms of number of models, macroseismic information, and processing. We selected five calibration 


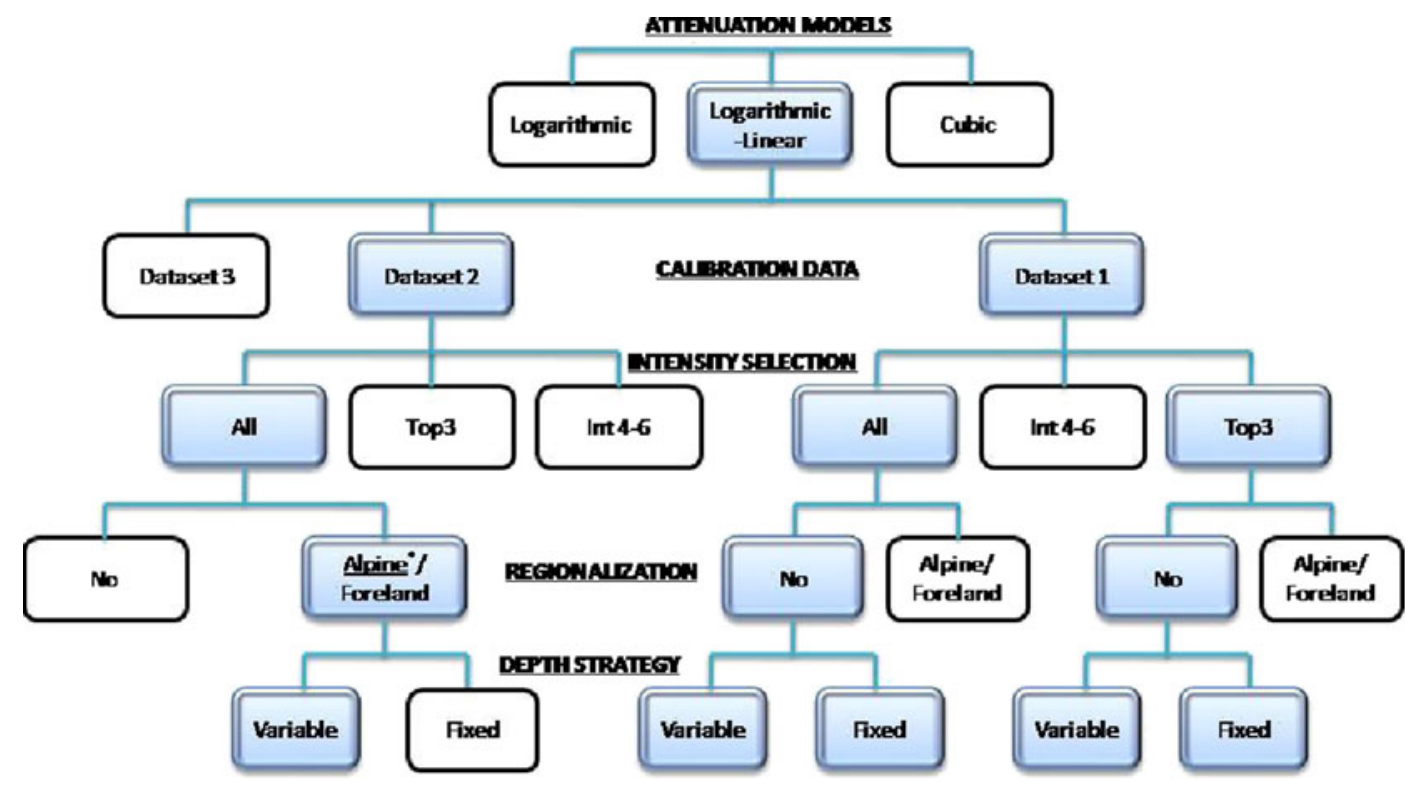

Fig. 3 Description of the different steps tested during the calibration process. Filled blue squares are those features that define the final calibration strategies applied in ECOS-09. (*)Only alpine regionalization has been selected

strategies through a continuous selection process, based on intermediate tests and results. The representation of the calibration procedure in Fig. 3 outlines the main steps and decisions for the selection of different calibrations strategies:

- Step 1: Evaluation of processing strategies for the intensity data: binning versus no-binning, evaluation of different distance weighting schemes. No binning was chosen due to its consistency with the application of the BW technique for the assessment of the macroseismic parameters.

- Step 2: Determination of the calibration coefficients of the different intensity attenuation models in Table 1, using (a) calibration datasets 1, 2, 3, with and without regionalization, and (b) all strategies concerning the intensity representation of calibration datasets: allint, top3, and int4-6. As performance metric to compare different intensity attenuation relationships (and parameters), relative variance reduction on non-binned data was used. Thus, the selection of an intensity attenuation model is independent of magnitudes.

- Step 3: Testing and selection of the best intensity attenuation models hereafter referred to as ECOS-09 models.
- Step 4: Assess calibration coefficients corresponding to the intensity to magnitude relation in Eq.2 for the selected ECOS-09 models and calibration strategies. The three different weighting schemes presented have been applied (weight the Intercept Intensity-Magnitude pair (a) equally for the events, (b) according to the number of intensity points contributing to the determination of its intercept intensity, and (c) according to the number and quality [information available to assess intensity] of the intensity observations contributing to the determination of an intercept intensity).

- Step 5: Testing and selection of the best intensity to magnitude relation for each of the calibration strategy of ECOS-09 models, including a reevaluation of the models by their ability to predict the instrumentally observed magni-

Fig. 4 Magnitude to $I_{30}$ relations for the five ECOS-09 calibration strategies. The three different lines in each plot correspond to the three weighting schemes tested for the relation. Black lines: each event with equal weight; green lines: number of intensity data points weighting; red lines: intensity data points quality weighting. A. Dataset 1, allint; fixed-depth; B. Dataset 1, allint; variable-depth (depth set here to $10 \mathrm{~km}$ ); C. Dataset 1, top3; fixed-depth; D. Dataset 1, top3; variable-depth (depth set here to $10 \mathrm{~km}$ ); E. Dataset 2, allint; alpine; variabledepth (depth set here to $10 \mathrm{~km}$ ) 

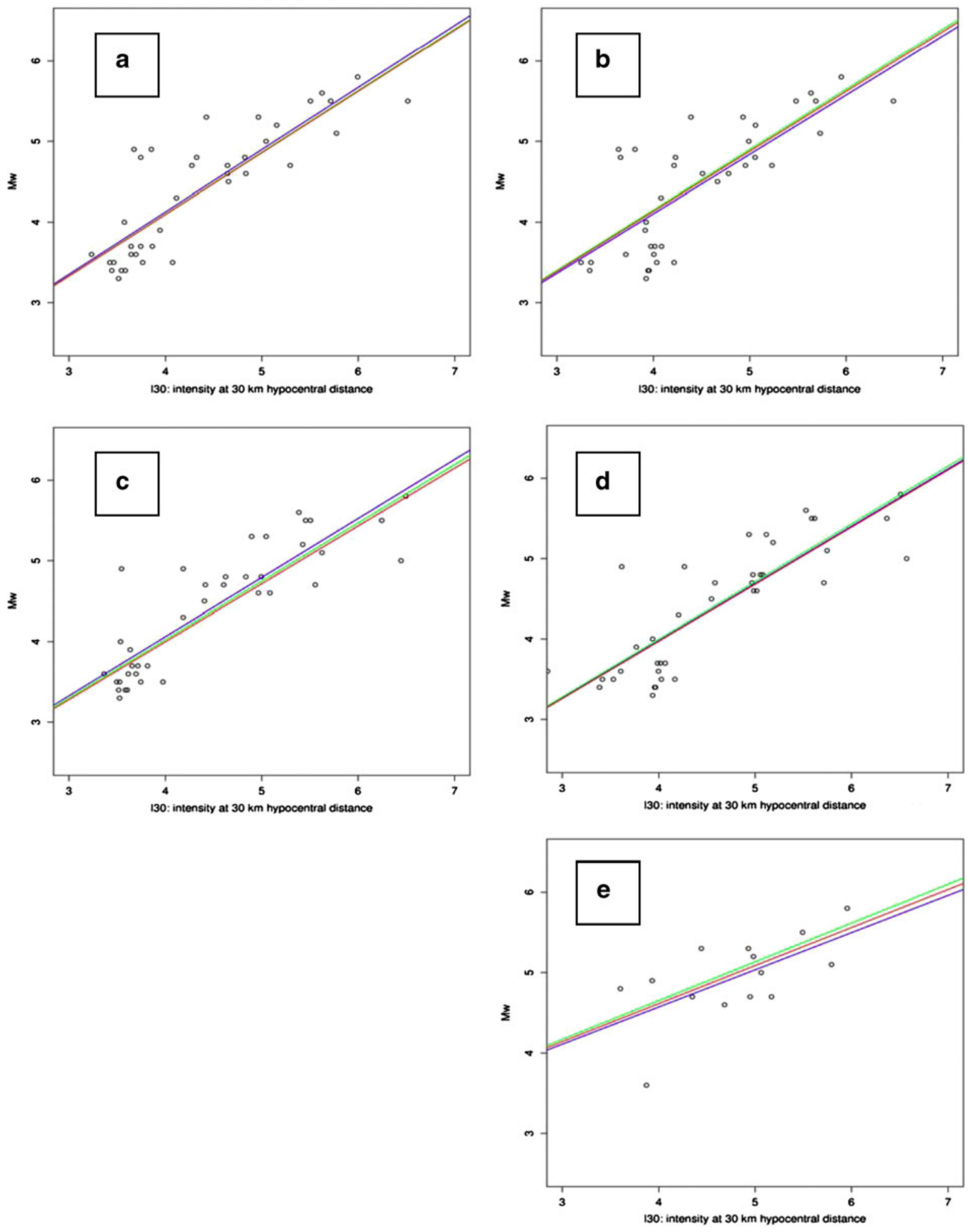
tudes. Metric was here the $R^{2}$ fitting parameter of the intercept intensity to magnitude regression model.

As result of steps 2 and 3 the following conclusions were drawn. It was found that the model with a logarithmic and linear attenuation term ( $R$, hypocentral distance) consistently showed the best performance when using the $R^{2}$ fitting parameter. This functional form of the attenuation model was therefore selected as ECOS-09 attenuation model:

$I-I_{\mathrm{sc}}=a \log \left(\frac{R}{h}\right)+b(R-h)$

The importance of the logarithmic and linear terms in the formulation is changing with the distance. The near-field behavior is controlled by the logarithmic term and the factor $a$, while the linear term and constant $b$ describes the far-field.

It was observed that the attenuation models calibrated with datasets 1 and 2 performed equally well. As it was expected, the attenuation model based on dataset 3 fitted better the data, due to the fact that the selection of events in dataset 3 was done from the fitting quality to the attenuation relation.

Regarding the use of regionalized data, calibration results with datasets 2 and 3 using only alpine events were rather different from the rest of foreland and non-regionalized attenuation models. We also found a different behavior when using all intensities (allint) of the events with intensities larger or equal to 3 , or only the three highest intensity levels (top 3) for each event. Step 4 showed that the events in dataset 3 do not cover a sufficient magnitude range to derive a reliable intensity to magnitude relation. For this reason, all calibrations with dataset 3 cannot be taken into consideration. As a conclusion to all these results, the following five calibration strategies have been selected and applied to the final assessment of macroseismic earthquake parameters, see Fig. 3: dataset 1, allint, fixed-depth; dataset 1, allint, variable-depth; dataset 1, top3, fixed-depth; dataset 1, top3, variable-depth; dataset 2, allint, alpine, variable-depth.

The influence of the three weighting schemes applied in the calibration of intensity to magnitude is shown in Fig. 4.

The final formulation of ECOS-09 attenuation model implemented in BW, see Eqs. 2 and 3 is:

$$
\begin{aligned}
M= & c_{1} I_{\mathrm{obs}}+c_{2} \operatorname{Ln}\left(\frac{R}{30}\right)+c_{3}(R-30)+c_{0} \\
& c_{0}=\beta ; c_{1}=\alpha ; c_{2}=-\alpha a ; c_{3}=-\alpha b
\end{aligned}
$$

Tables 2, 3, and 4 list the values of all calibrated coefficients of the five final calibration strategies.

Focal depth is the least constrained parameter and our main question is, if a reliable depth for a particular event can be estimated. A first attempt to focal determination has been carried out during the calibration exercise, by assigning a best fitting depth to each calibration event during the iteration process

\begin{tabular}{|c|c|c|c|c|c|c|}
\hline \multicolumn{4}{|l|}{ Strategy } & \multicolumn{3}{|l|}{ Calibrated coefficients } \\
\hline $\begin{array}{l}\text { Calibration } \\
\text { dataset }\end{array}$ & $\begin{array}{l}\text { Intensity } \\
\text { representation }\end{array}$ & Regionalization & Depth & $\begin{array}{l}\text { Logarithmic } \\
\text { coefficient }(a)\end{array}$ & Linear coefficient $(b)$ & SD \\
\hline 1 & All intensity levels ${ }^{\mathrm{a}}$ & - & $\begin{array}{l}\text { Fixed depth } \\
\quad(h=10 \mathrm{~km})\end{array}$ & $-0.67755 \pm 0.02636$ & $-0.00174 \pm 0.0006007$ & 0.4073 \\
\hline 1 & $\begin{array}{l}\text { Three highest } \\
\text { intensity levels }\end{array}$ & - & $\begin{array}{l}\text { Fixed depth } \\
\quad(h=10 \mathrm{~km})\end{array}$ & $-0.4834 \pm 0.02589$ & $-0.00179 \pm 0.0006097$ & 0.3647 \\
\hline 1 & All intensity levels ${ }^{\mathrm{a}}$ & - & $\begin{array}{l}\text { Variable depth } \\
\qquad(h=3-25 \mathrm{~km})\end{array}$ & $-0.69182 \pm 0.008803$ & $-0.00084 \pm 0.0002967$ & 0.3897 \\
\hline 1 & $\begin{array}{l}\text { Three highest } \\
\text { intensity levels }\end{array}$ & - & $\begin{array}{l}\text { Variable depth } \\
\qquad(h=3-25 \mathrm{~km})\end{array}$ & $-0.50945 \pm 0.008224$ & $-0.00192 \pm 0.0002836$ & 0.3556 \\
\hline 2 & All intensity levels ${ }^{\mathrm{a}}$ & Alpine & $\begin{array}{l}\text { Variable depth } \\
\qquad(h=3-25 \mathrm{~km})\end{array}$ & $-1.07853 \pm 0.01952$ & $0.00414 \pm 0.000630$ & 0.4226 \\
\hline
\end{tabular}

Table 2 Calibrated coefficients of ECOS-09 intensity attenuation model for the selected strategies

\footnotetext{
${ }^{\mathrm{a}}$ Intensity III and larger
} 


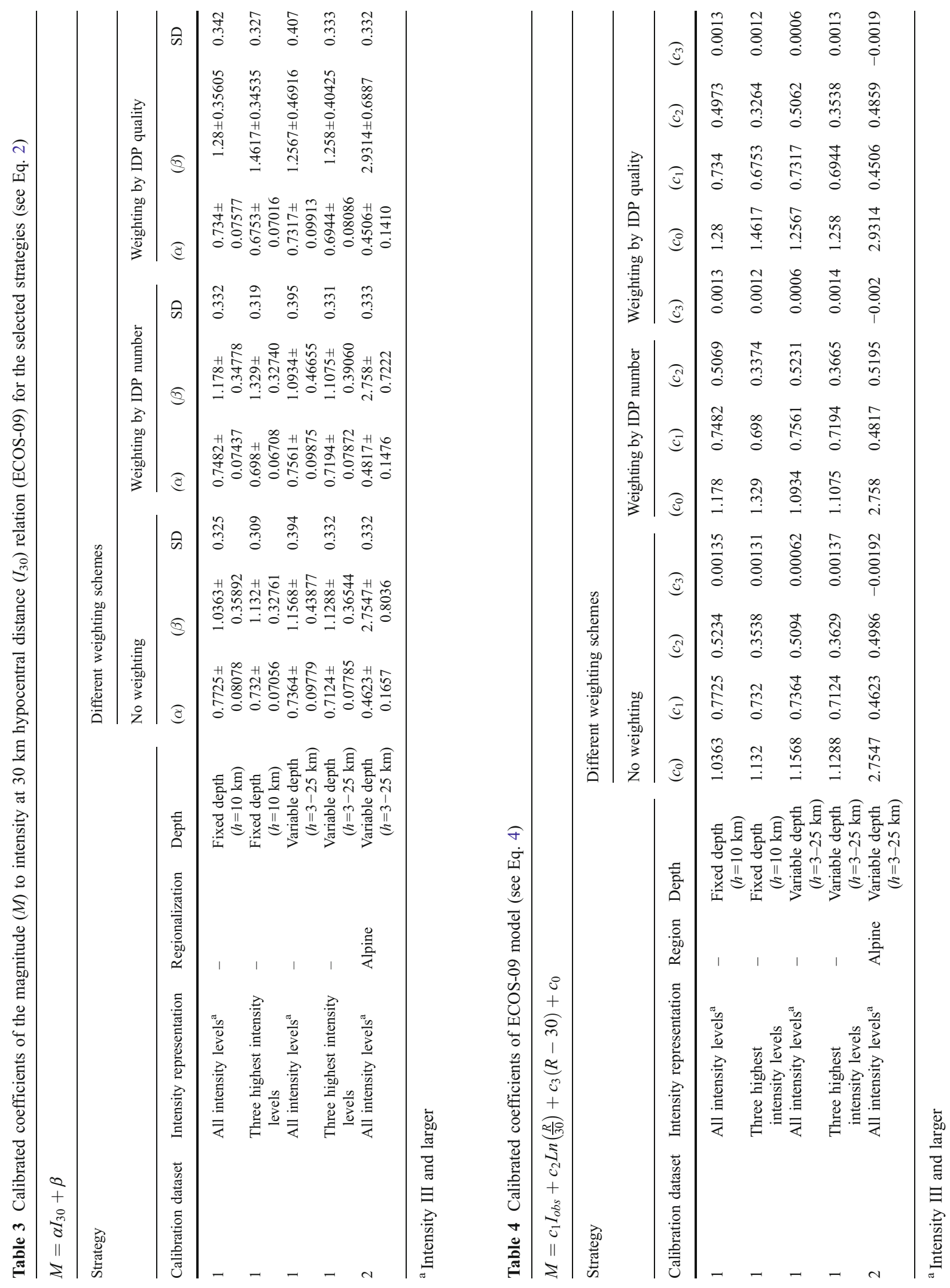


of the regression performed for the variable-depth strategy.

Figure 5 shows a sample of the depths derived for dataset 1 during the calibration. A trend with shallower events in the alpine area and deeper events in the foreland area can be observed. This feature is in general agreement with our knowledge of the depth distribution of earthquakes from the instrumental period since 1975. It encouraged us to address the estimation of depth for particular events during the assessment of macroseismic parameters. It has to be taken into account that there is almost no quantitative depth information of the larger events in our calibration dataset, and therefore derived depths cannot be compared with instrumentally determined depths.

\subsection{Validation of the calibration of ECOS-09 model}

A first validation of the calibration was carried out by comparing assessed macroseismic magnitudes with BW to known instrumental magnitudes. This was done for a set of events with $M_{w}$ (bestmag). In the application of BW technique, the assessment of the macroseismic location depends critically on the quantity and quality of the intensity data points, along with their distribution relative to the earthquake source location. Furthermore, distance weighting may lead to bogus RMS minima if the search area stretches to areas too far away from the relevant part of the macroseismic field. For this reason, and in order to avoid fake minimum residuals, special care has been taken when setting and scaling the grid search area of trial epicenters, with the dimensions of the area delimited by the macroseismic field.

We have used available historical information in order to constrain the epicenter location and therefore the search area. In the cases where a previous ECOS-02 catalogue location is known, the search area has been centered on this point and extended by a total width of $75 \mathrm{~km}$ in each direction. In the case where there is no certain catalogue location, the search area was given by
Fig. 5 Depths assigned to events of calibration dataset 1 during the calibration process following strategy: allint; variable-depth

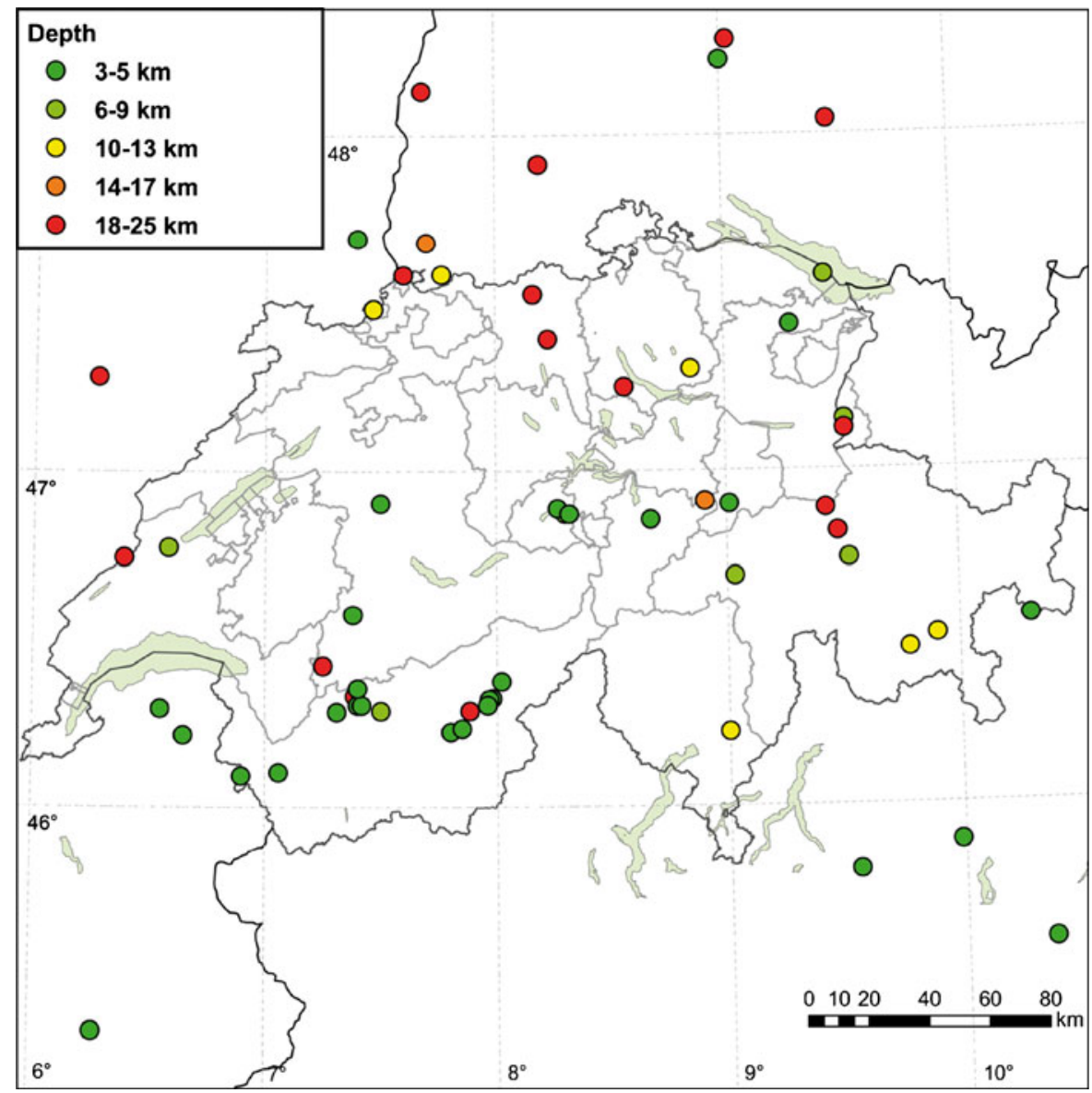


the bounding box containing all intensity data points of the two intensity classes below the maximum intensity. The macroseismic magnitude was assessed at the original catalogue location (hereafter $M_{\text {cat }}$ ), at the minimum magnitude location $\left(M_{\mathrm{mag}}\right)$ and the mini-

a

Törbel 1855.07.25

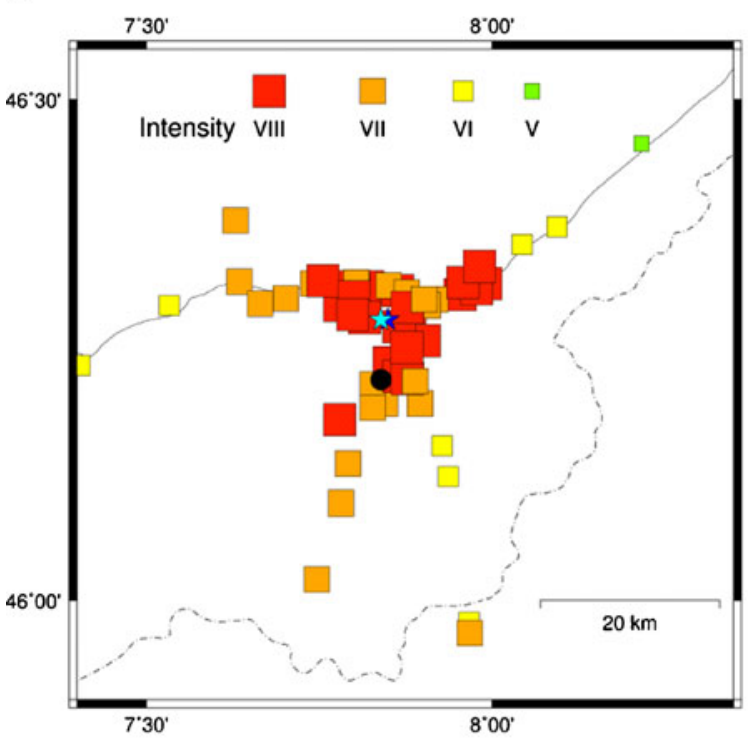

b

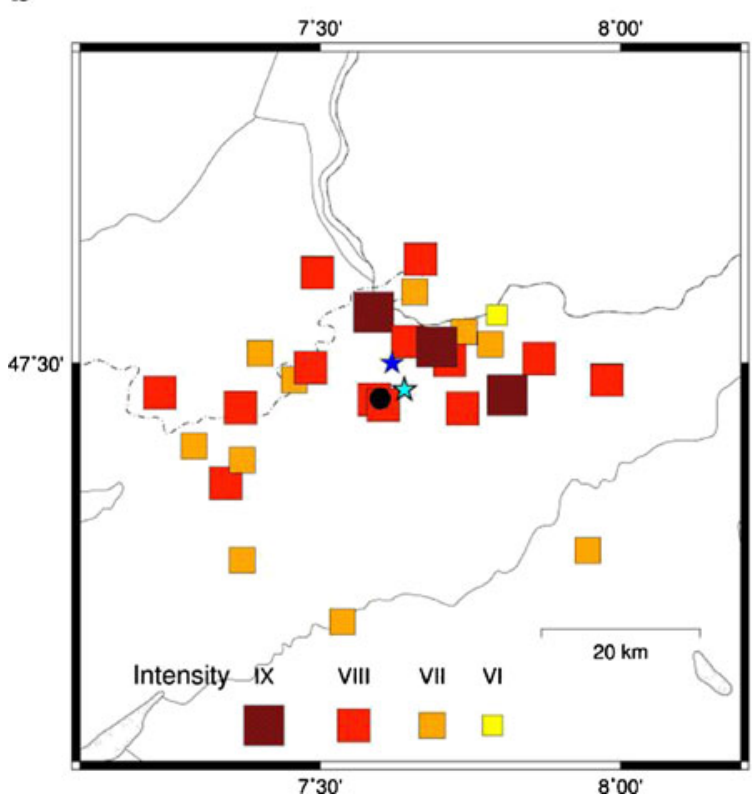

Fig. 6 Display of BW assessment results and the macroseismic field of two events: a Törbel event (1855.07.25). $M_{\text {cat }}=6.23 ; M_{\mathrm{mag}}=6.2 ; M_{\mathrm{RMS}}=6.2$. Strategy used: allint, variable-depth $(h=10 \mathrm{~km})$ b Basel event (1356.10.18). $M_{\text {cat }}=6.57$; mum RMS location ( $\left.M_{\mathrm{RMS}}\right)$. In Fig. 6 are displayed the results of BW for two events, the 1855 Valais event and the 1356 Basel earthquake.

Figure 7 shows the comparison between assessed macroseismic magnitude and instrumental magnitude.

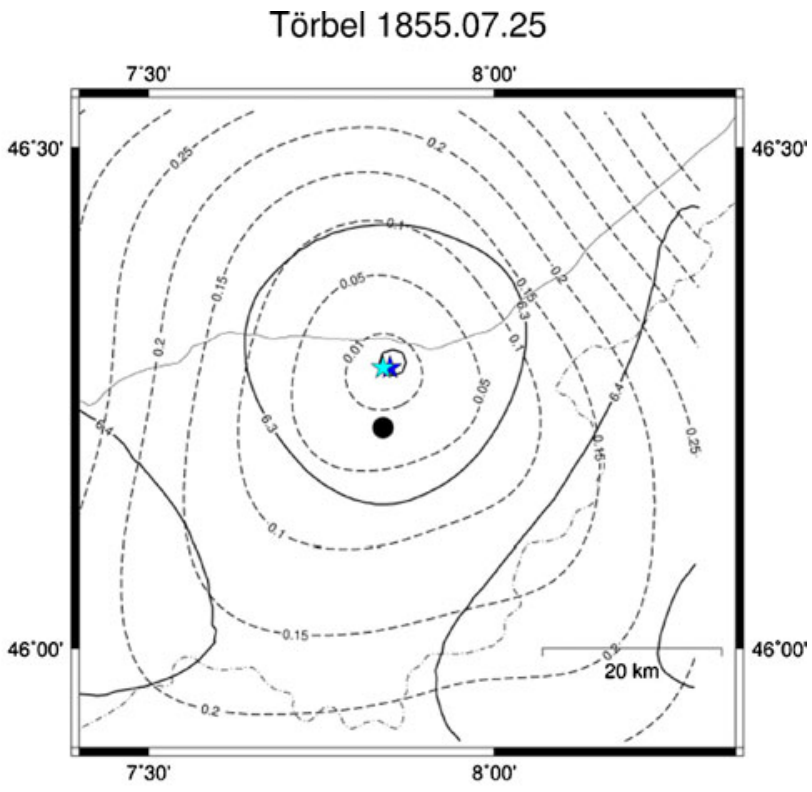

Basel 1356.10.18

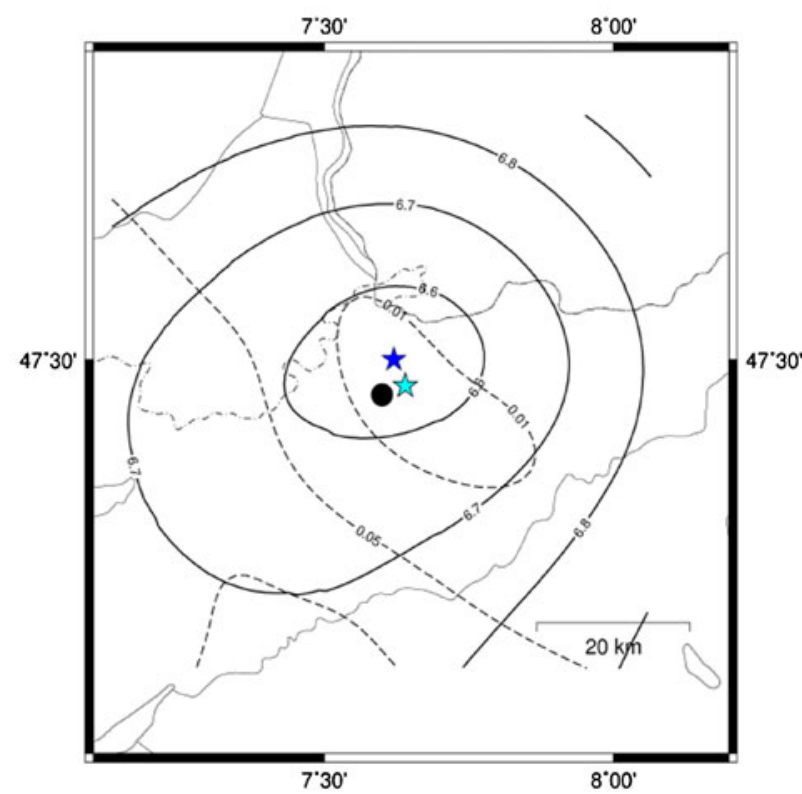

$M_{\text {mag }}=6.56 ; M_{\mathrm{RMS}}=6.57$. Strategy used: top3, fixed-depth. Solid contours: magnitude values; dashed contours RMS values; black circle: catalogue location; dark blue star: minimum magnitude location; light blue star: minimum RMS location 

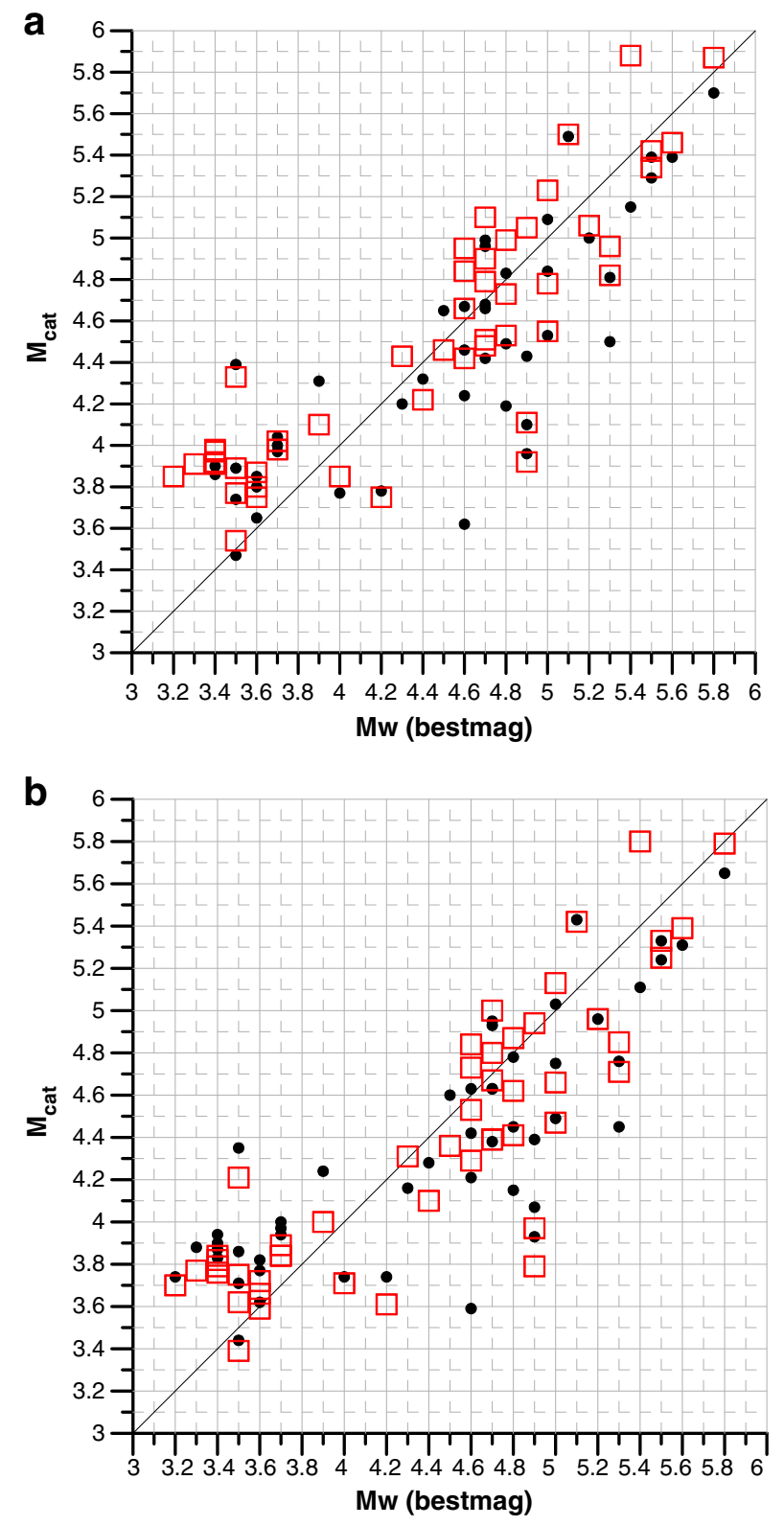

Fig. 7 Estimated magnitudes at the epicenter locations $\left(M_{\text {cat }}\right)$ for subsets of events of calibration dataset 1 with instrumental magnitude $\left(M_{w}\right.$ (bestmag) $)$. In the magnitude to intensity calibration, see Eq.2; intensity data points are weighted by their quality. a (filled circles) allint, fixed-depth strategy;

The performance of the BW method is good, especially for events with magnitudes larger than about magnitude 4, which is also the magnitude range of particular interest for the historical period of the catalogue.

A second calibration exercise has been carried out in parallel applying the Boxer method (Gasperini et al. 1999; Fäh et al 2011). The earthquake parameters

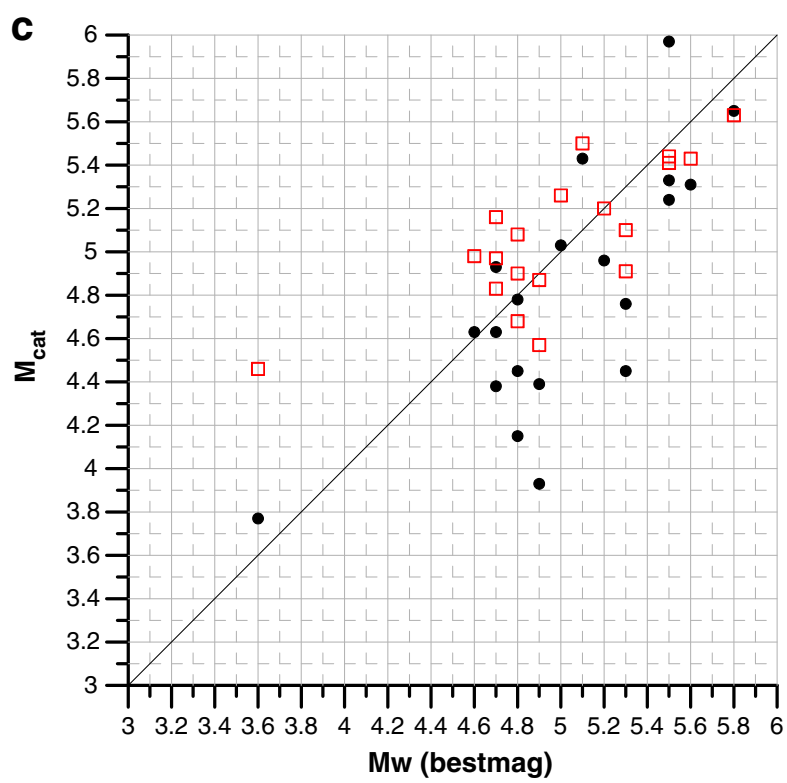

Mw (bestmag) (open squares) top3; fixed-depth strategy. b (filled circles) allint, variable-depth strategy $(h=10 \mathrm{~km})$; (open squares) top3, variable-depth strategy $(h=10 \mathrm{~km})$. c (filled circles) allint, variable-depth strategy $(h=10 \mathrm{~km})$; (open squares) allint, alpine, variable-depth strategy $(h=10 \mathrm{~km})$

assessed are epicenter location and magnitude. This calibration served as a cross-check of ECOS-09 $\mathrm{BW}$ results. In a first step, the Boxer method was calibrated with a selected dataset of Swiss events of the twentieth century for which macroseismic data and moment magnitudes were available. This calibration was then tested. In a second step, other existing calibration 
coefficients for the Boxer method were applied to the same Swiss dataset. One of these calibrations was derived from a selected dataset of Swiss events during the NERIES project (Gómez Capera et al. 2009), while other calibrations were derived from Italian data during the NERIES project and a national research project. Within the magnitude range of Swiss historical events, there is a need to assess parameters for earthquakes larger than the events in the Swiss calibration dataset. Basically, the Swiss calibration dataset does not provide reliable coefficients for the Boxer code above a moment magnitude of about 5.5, or for intensity 7 or larger. Furthermore, the extrapolation of the calibration method applied in ECOS-09 cannot be controlled for magnitudes larger than about 6.0. Therefore, independent coefficients that cover earthquakes with magnitudes above this limit were included in our study using the Boxer code. This has been achieved using coefficients for the Boxer code from the NERIES project and an Italian study. Finally, all calibration coefficients have been tested, and different strategies were proposed including hybrid strategies that combine the lowintensity Swiss calibration with Italian high-intensity calibration coefficients.

From the suite of possible calibration coefficients for the Boxer method, a selection was applied to assess macroseismic location and magnitude of some historical events; see Table 5. Since all the calibration procedures were validated, the assessment with Boxer provides a range of magnitude for each event. With the help of the Boxer method, we can confirm our assessment of magnitudes based on the $B W$ method within the limits of the calibration methods.

In Fig. 8, ECOS-09 model (Eq.4) has been compared with a revised ECOS-02 model and formulations derived for central Europe by Bakun and Scotti

Table 5 Comparison of magnitudes derived from different approaches for a selection of event

\begin{tabular}{|c|c|c|c|c|c|c|c|c|c|c|}
\hline Year & Month & Day & Hour & $\begin{array}{l}\text { Event Name in } \\
\text { ECOS- } 02\end{array}$ & $M_{\mathrm{w}}\left(\right.$ bestmag) ${ }^{\mathrm{a}}$ & $\begin{array}{l}M_{\mathrm{w}} \\
\text { ECOS- } 02^{\mathrm{b}}\end{array}$ & $\begin{array}{l}\text { Revised } M_{\mathrm{w}} \\
\text { ECOS- } 02^{\mathrm{c}}\end{array}$ & $\begin{array}{l}\text { Range } M_{\mathrm{w}}{ }^{\mathrm{d}} \\
\text { Boxer }\end{array}$ & $\begin{array}{l}M_{\mathrm{w}} \text { from BW } \\
\text { method }^{\mathrm{e}}\end{array}$ & $\begin{array}{l}M_{\mathrm{w}} \\
\text { ECOS- } 09^{\mathrm{f}}\end{array}$ \\
\hline 1295 & Sept & 03 & 00 & Churwalden & - & 6.5 & 6.2 & $6.0-6.5$ & 6.2 & 6.2 \\
\hline 1356 & Oct & 18 & 21 & Basel & - & 6.9 & 6.7 & $6.3-6.9$ & 6.6 & 6.6 \\
\hline 1584 & Mar & 11 & 11 & Aigle & - & 6.4 & 6.1 & $5.6-6.1$ & 5.9 & 5.9 \\
\hline 1601 & Sept & 18 & 1 & Unterwalden & - & 6.2 & 5.9 & $5.9-6.2$ & 5.9 & 5.9 \\
\hline 1685 & Mar & 8 & 19 & Mittelwallis & - & 6.1 & 5.9 & $5.4-5.8$ & 5.3 & 5.3 \\
\hline 1755 & Dec & 9 & 13 & Brig-Naters & - & 6.1 & 5.9 & $5.8-6.1$ & 5.7 & 5.7 \\
\hline 1770 & Mar & 20 & 15 & Château-d'Oex & - & 5.7 & 5.5 & $4.9-5.4$ & 5.2 & 5.2 \\
\hline 1855 & Jul & 25 & 11 & Törbel & - & 6.4 & 6.1 & $5.9-6.1$ & 6.2 & 6.2 \\
\hline 1905 & Dec & 25 & 17 & Domat-Ems & 4.7 & 4.8 & 4.7 & $4.5-5.1$ & 4.8 & 4.7 \\
\hline 1905 & Dec & 26 & 0 & Tamins & - & 5.1 & 5.0 & $4.6-5.2$ & 4.7 & 4.7 \\
\hline 1913 & Jul & 20 & 12 & Ebingen & - & 5.2 & 5.1 & $4.7-5.3$ & 5.0 & $5.2^{\mathrm{g}}$ \\
\hline 1929 & Mar & 1 & 10 & Bioley-Magnoux & 5.0 & 5.3 & 5.0 & $4.3-5.1$ & 4.7 & 5.0 \\
\hline 1946 & Jan & 25 & 17 & Ayent & 5.8 & 6.1 & 5.8 & $5.8-6.0$ & 5.7 & 5.8 \\
\hline 1946 & May & 30 & 3 & Ayent & 5.5 & 6.0 & 5.5 & $5.2-5.6$ & 5.4 & 5.5 \\
\hline 1978 & Sept & 3 & 5 & Ebingen & 5.5 & 5.15 & 5.5 & $5.1-5.6$ & 5.3 & 5.5 \\
\hline 1991 & Nov & 20 & 1 & Vaz/GR & 4.7 & 4.6 & 4.7 & $4.7-5.3$ & 4.6 & 4.7 \\
\hline
\end{tabular}

The event names refer to the names in the 2002 catalogue. They changed in ECOS-09

${ }^{\mathrm{a}} M_{w}$ (bestmag): $M_{\mathrm{w}}$ derived from instrumental recordings by Bernardi et al. (2005)

${ }^{\mathrm{b}} M_{\mathrm{w}}$ in the ECOS-02 catalogue

${ }^{\mathrm{c}}$ Revised $M_{\mathrm{w}}$ due to changes in magnitudes of the calibration events used for ECOS-02 (see Table 6)

${ }^{\mathrm{d}}$ Magnitude range obtained with the Boxer method using different strategies

${ }^{\mathrm{e}} M_{\mathrm{w}}$ estimated with the BW method for ECOS-09

${ }^{\mathrm{f}} M_{\mathrm{w}}$ in the ECOS-09 catalogue

${ }^{\mathrm{g}}$ Magnitude from foreign catalogue 
Fig. 8 Intensity attenuation models included in Table 6 and ECOS-09 models (Eq.4). The plots correspond to an event $M=5.0$ at $10 \mathrm{~km}$ depth. The ECOS-02 shallow event relations are not applicable for events at $10 \mathrm{~km}$ depth

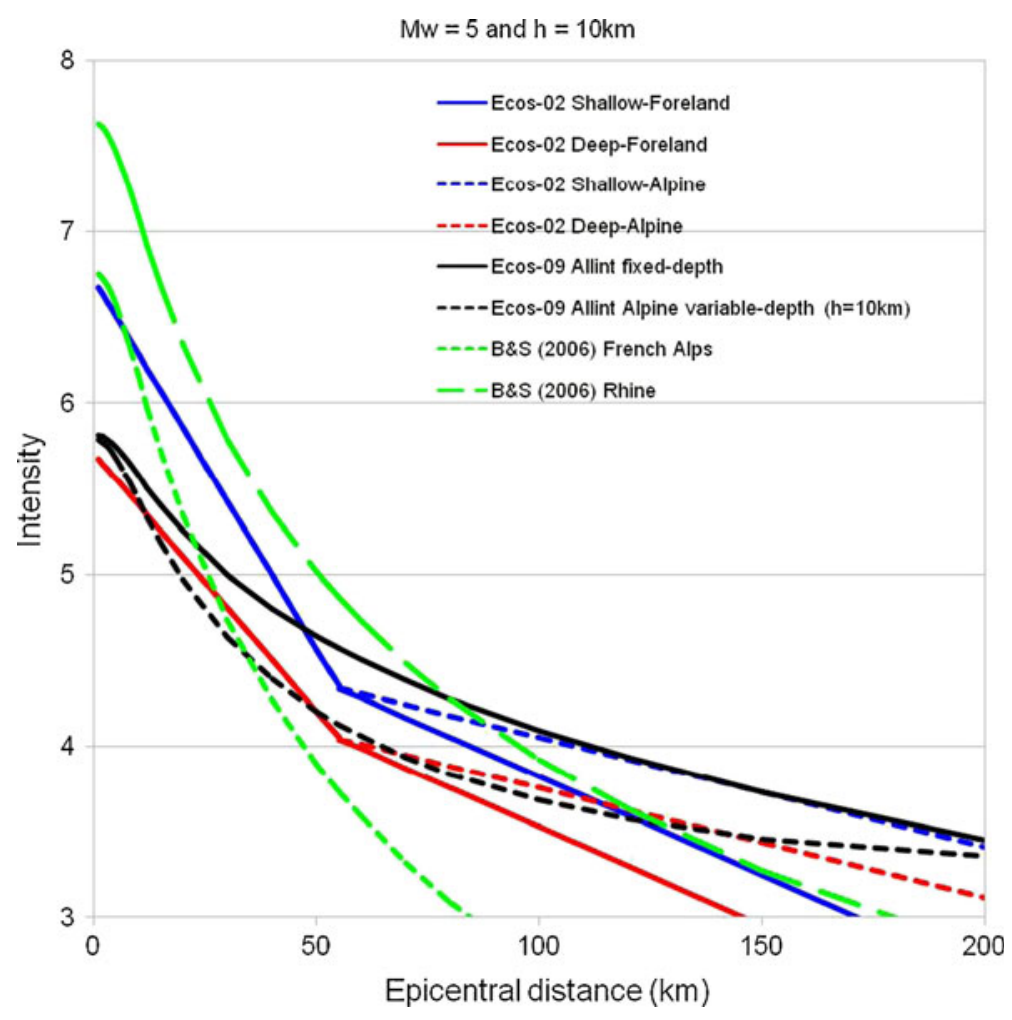

(2006). These models are presented in Table 6. ECOS02 proposed attenuation relations for sources in the Alpine and Foreland regions; both relations were developed for shallow events and deep events. All these attenuation relations have a bilinear form with a hinge around $55 \mathrm{~km}$ epicentral distance. Magnitudes of calibration events changed after 2002 mostly due to the work performed by Braunmiller et al. (2005) and Bernardi et al. (2005). Therefore, the revised
ECOS-02 model includes the revision of the intercept-intensity to magnitude relation using the new moment magnitudes published in Bernardi et al. (2005). We have also included the models developed in Bakun and Scotti (2006) for the French Alps and the Rhine region in the comparison. These models have been calibrated with seven and six events respectively, and using MSK intensity values and the SisFrance (2003) database for most events.

Table 6 Different attenuation models compared with ECOS-09 model

\begin{tabular}{|c|c|c|}
\hline References & Distance & Intensity attenuation models \\
\hline ECOS-02 revised ${ }^{\mathrm{a}}$ shallow events & $\mathrm{Up}$ to $55 \mathrm{~km}$ & $I_{\exp }=-0.9079+1.5248 M_{w}-0.043 D$ \\
\hline ECOS-02 revised ${ }^{\mathrm{a}}$ deep events & $\mathrm{Up}$ to $55 \mathrm{~km}$ & $I_{\exp }=-2.8941+1.7196 M_{w}-0.030 D$ \\
\hline ECOS-02 revised $^{\mathrm{a}}$ shallow foreland events & $55-200 \mathrm{~km}$ & $I_{\exp }=-2.6539+1.5248 M_{w}-0.0115 D$ \\
\hline ECOS-02 revised ${ }^{\mathrm{a}}$ shallow alpine events & $55-200 \mathrm{~km}$ & $I_{\exp }=-2.9339+1.5248 M_{w}-0.0064 D$ \\
\hline ECOS-02 revised ${ }^{\mathrm{a}}$ deep foreland events & $55-200 \mathrm{~km}$ & $I_{\exp }=-3.9241+1.7196 M_{w}-0.0115 D$ \\
\hline ECOS-02 revised ${ }^{\mathrm{a}}$ deep alpine events & $55-200 \mathrm{~km}$ & $I_{\exp }=-4.2041+1.7196 M_{w}-0.0064 D$ \\
\hline Bakun and Scotti (2006) Rhine & $10-250 \mathrm{~km}$ & $I_{\exp }=5.0+1.27 M_{w}-3.71 \log (R)$ \\
\hline Bakun and Scotti (2006) French Alps & $10-110 \mathrm{~km}$ & $I_{\exp }=4.46+1.27 M_{w}-4.05 \log (R)$ \\
\hline
\end{tabular}

$I_{\text {exp }}$ intensity at the site, $D$ epicentral distance, $R$ hypocentral distance

${ }^{\text {a }}$ For magnitudes above 5.5, only the relation for deep events is used 
From Fig. 8, we conclude that ECOS-02 revised for deep events is very similar to the ECOS-09 attenuation models. ECOS-02 curves for shallow events, as expected, present an offset in the nearfield because these relations are not applicable to events with a source depth of $10 \mathrm{~km}$. Bakun and Scotti (2006) models, however, also show this offset, which would underestimate magnitudes in our assessment. It has to be noted that the calibration in ECOS-02 was performed based on few events only. The lower rate of decay in the far-field in ECOS-09 in comparison of ECOS-02 revised is due to the difference in the formulation and the processing of the macroseismic field of the calibration events (e.g., intensities less than 3 were removed for ECOS-09, a quadratic weighting scheme has been applied, etc.).

\section{Estimation of macroseismic earthquake parameters of historical events in ECOS-09}

The following strategy has been applied for the assessment of earthquake parameters of historical events in ECOS-09 using the $B W$ method:

1. Four calibrated non-regional attenuation models and one Alpine model were applied. Two models are for fixed depth at $10 \mathrm{~km}$, while three strategies are with variable depth. The four non-regional relations are derived from the largest calibration dataset (dataset 1), and the Alpine relation is derived from a smaller one (dataset 2; calibrated coefficients are given in Tables 2 to 4).

2. Using the $B W$ method, the macroseismic magnitude was assessed at the original catalogue location, at the location of the minimum magnitude and the minimum magnitude root mean square (RMS). Applying the different attenuation models resulted in some scatter of the source location based on the minimum magnitude and on the minimum RMS. In most cases, the ECOS-02 location is close to the derived locations of the minimum RMS. The epicenter location in the ECOS-02 catalogue was determined taking into account the available historical information and BW location, and as long as there is no new historical evidence that this epicenter is incorrect, or that the location is different to the BW locations based on minimum RMS, the location was not altered. The RMS as function of depth at the epicenter location was then derived. If the RMS curve shows a relevant minimum, the depth and corresponding magnitude were estimated (see an example in Figs. 9 and 10). For events that have new historical evidence or a new macroseismic dataset, a full re-assessment of the epicenter location was performed using all information.
Fig. 9 Intensity field of the 1774.09.10 Altdorf event. Black circle: catalogue location; dark blue star: minimum magnitude location; light blue star: minimum RMS location. The catalogue location corresponds well to the minimum RMS location

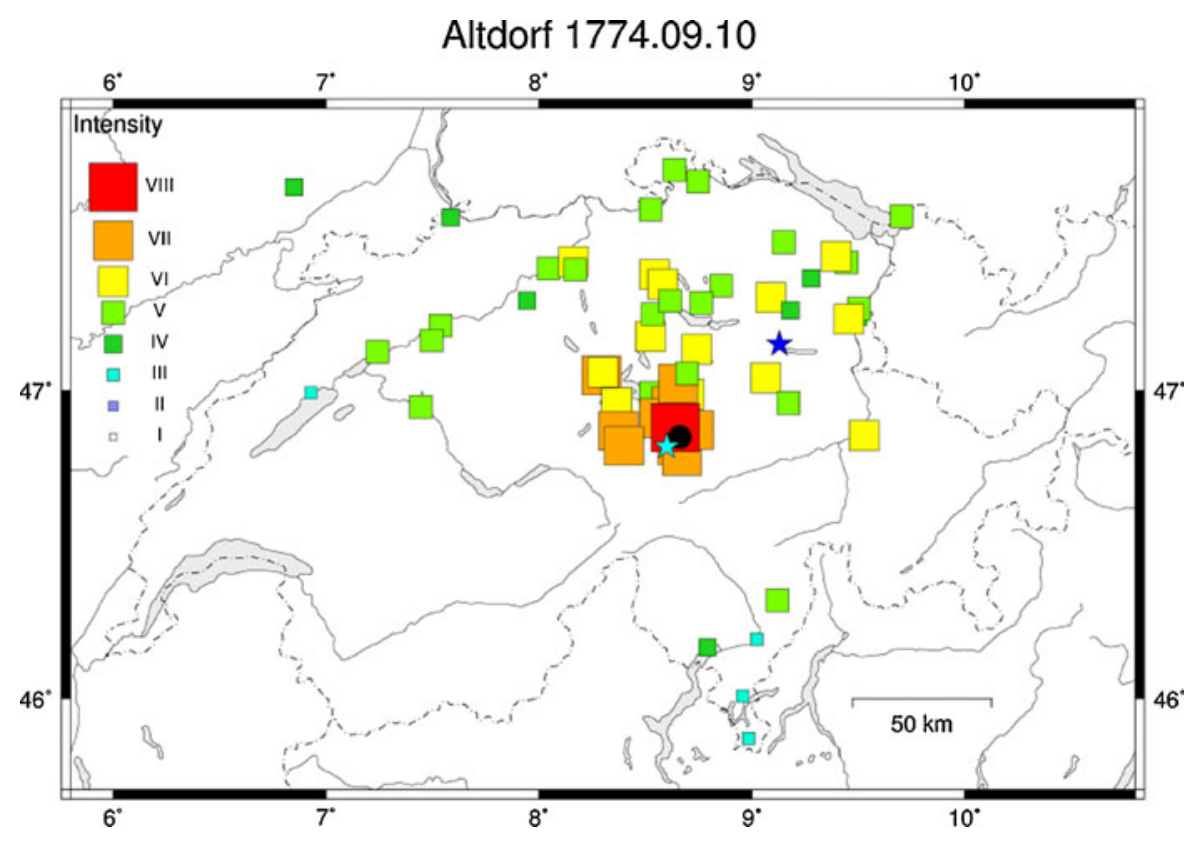


Fig. 10 After the epicenter is defined, a the magnitude as function of depth and $\mathbf{b}$ the RMS as a function of depth are computed. The figures refer to the 1774.09.10 Altdorf event (Fig. 9) and the strategies: dataset 1 , allint, and top 3 for variable-depth; dataset 2 , alpine, allint variable-depth . For this event, the assigned depth is $8 \mathrm{~km}$, and the corresponding magnitude is 5.7

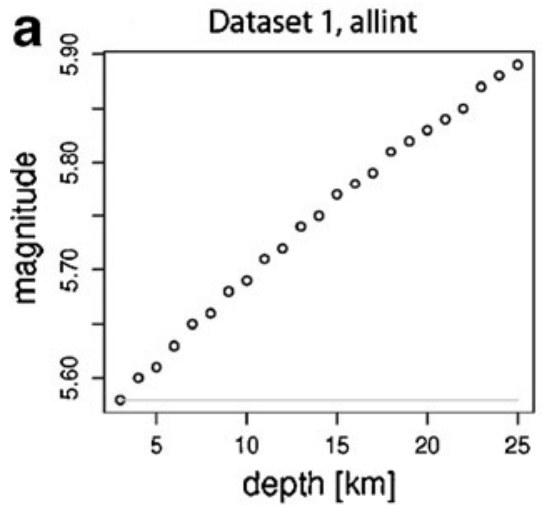

Dataset 2, alpine, allint
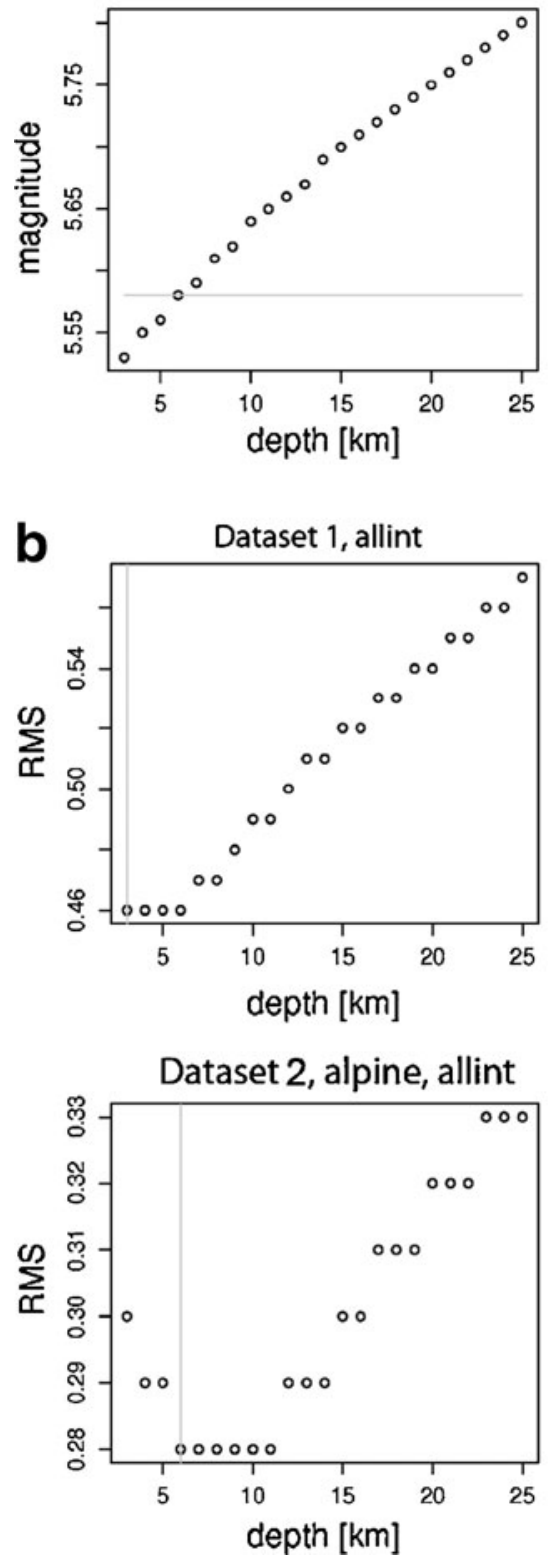
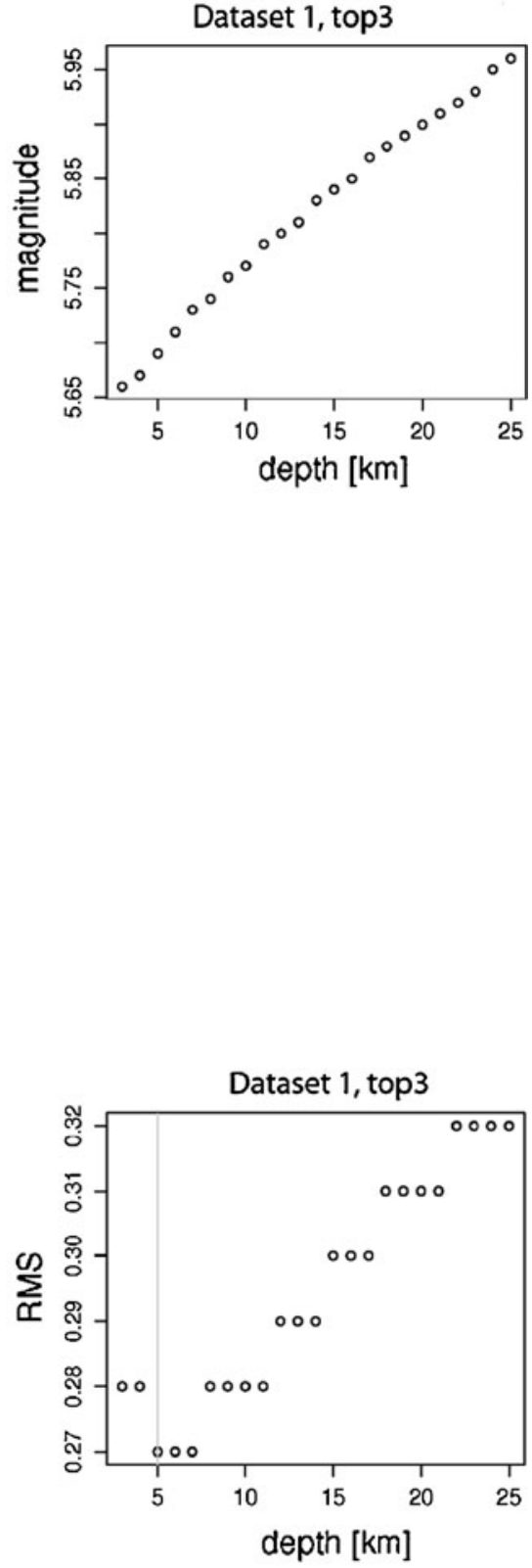
Fig. 11 Control plot: After the epicenter is defined, the macroseismic field is plotted as a function of epicentral distance, overlaying the theoretical curves by assuming different depth levels (at 3, 6, 10, 15, and 20km), and using the derived magnitudes at the corresponding depth. The figures refer to the 1774.09.10 Altdorf event (Fig. 9), and the strategies: dataset 1, allint (top figure), and top3 (figure in the middle); dataset 2, alpine, allint (figure on the bottom). The blue curves correspond to the attenuation model assuming fixed-depth at $10 \mathrm{~km}$

3. In order to better control the depth selection, the macroseismic field of each event and the corresponding intensity curves have been plotted as function of epicentral distance (Fig. 11). The curves have been drawn for different depth levels and using the derived magnitudes at the corresponding depth. The final decision on the event's magnitude and depth is made by including information from the RMS plots (horizontally as well as vertically) and taking into account the possibility of poor performance of one of the calibration strategies. If no clear decision of depth was possible, the magnitude corresponding to a depth of $10 \mathrm{~km}$ was taken. In this case, no depth is assigned to the event. The magnitude is defined by the median of magnitudes from the strategies that perform well and are valid for this case (Alpine only for alpine events, only depth strategies in case a depth is assigned to the events). Finally, each event was compared with events of similar magnitude, and a few magnitudes needed adjustments for those cases in which the event had obviously too high or too low magnitude simply caused by very sparse macroseismic fields. In such cases, the calibration procedure failed, and the data points from the events were compared with events of similar size with more intensity data points to assign a reasonable magnitude.

\section{Uncertainties in the assessment of location and magnitude}

Each stage of the assessment of macroseismic parameters has an inherent random and model uncertainty, which propagates through the process and determines the total uncertainty in the estimated earthquake parameters. In the following, we will argue that an exact statistical evaluation of errors is not achievable, due to a lack of independent information. Nevertheless, we are able to quantitatively assess bounds of the uncertainties for magnitudes and locations.
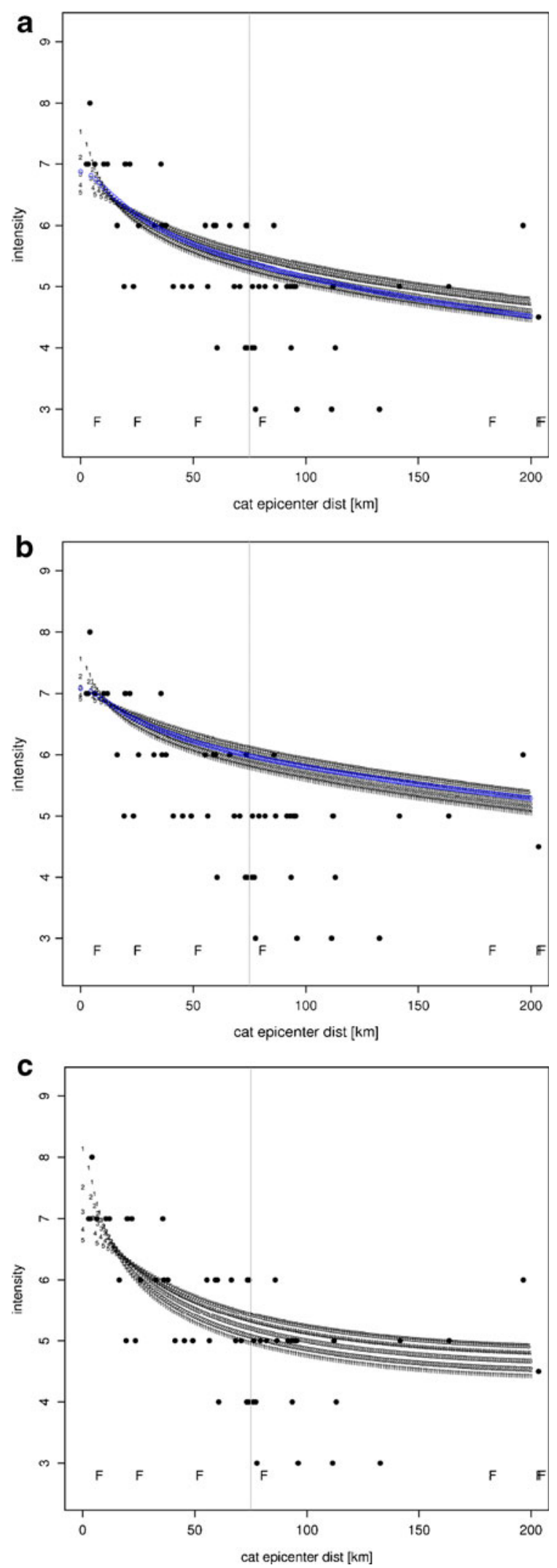
Two of the main sources of uncertainty are related to the macroseismic field. The first stems from the intensity range assigned to a single intensity data point (the range of intensities consistent with the reported effects of that site). Each intensity data point has a most probable intensity $I_{\mathrm{w}}$, and a minimum intensity $I_{\min }$ and maximum intensity $I_{\max } . I_{\min }$ and $I_{\max }$ define the possible intensity range. The second source of errors is the distribution of data in the macroseismic field. Problems might arise from irregular azimuthal coverage due to national borders, gaps in historical information, and variability of the number of intensity data points due to factors such as population density. A further source of uncertainty has a methodological origin. Calibration models, both the attenuation model and the standardized intensity to magnitude relation, also introduce uncertainties. They rely on calibration datasets with more or less reliable moment magnitudes and irregular macroseismic fields. An uncertainty range is provided for most of the $M_{w}$ (bestmag), but not all. Furthermore, these values do not cover the full uncertainty related to the methods applied by Bernardi et al. (2005) and Braunmiller et al. (2005). Some of the calibration events have an inhomogeneous geographical distribution of intensity data points; depth is unknown for most of the calibration events, and epicenter location uncertainty of the calibration events cannot be assessed. We have limited control on the contribution of these uncertainties to the overall uncertainty. Nevertheless, we have studied some of aspects of this problem.

The bootstrap statistical method is a procedure that deals with the problems arising from the incompleteness of the intensity field. The sampling with replacement can provide a set of different resampled macroseismic fields, such that the uncertainty of the magnitude and location is represented by the distribution of the locations and magnitudes obtained from these resampled datasets (see Bakun and Scotti 2006). We applied bootstrap to a selection of events with dense and sparse intensity fields. In a first step, for each event 1,000 intensity sets were prepared in a standard bootstrap with replacement process, providing in each sample and for strategies dataset 1 allint, dataset 1 top 3 , and dataset 2 allint alpine, the same number of intensity data point sites as in the original macroseismic field. In a second step, we resampled each individual intensity assessment in order to reflect its uncertainty. Based on the three intensity values $I_{\mathrm{w}}, I_{\min }$, or $I_{\max }$, we reassigned intensity applying a probability model with the following rule set:

\begin{tabular}{|c|c|c|}
\hline & Intensity & Probability \\
\hline$I_{\mathrm{w}} \neq I_{\min }$ and $I_{\mathrm{w}} \neq I_{\max }$ & $I_{\mathrm{w}}$ & $50 \%$ \\
\hline$I_{\min }=I_{\mathrm{W}}-1$ & $I_{\min }$ & $25 \%$ \\
\hline$I_{\max }=I_{\mathrm{w}}+1$ & $I_{\max }$ & $25 \%$ \\
\hline \multirow{2}{*}{$I_{\mathrm{w}} \neq I_{\min }$ and $I_{\mathrm{w}} \neq I_{\mathrm{max}}$} & $I_{\mathrm{w}}$ & $50 \%$ \\
\hline & $I_{\mathrm{w}-1}$ & $18 \%$ \\
\hline$I_{\min }=I_{\mathrm{w}}-2$ & $I_{\min }$ & $7 \%$ \\
\hline \multirow{2}{*}{$I_{\max }=I_{\mathrm{w}}+2$} & $I_{\mathrm{w}+1}$ & $18 \%$ \\
\hline & $I_{\max }$ & $7 \%$ \\
\hline \multirow{2}{*}{$I_{\mathrm{w}}=I_{\min }$} & $I_{\min }$ & $75 \%$ \\
\hline & $I_{\max }$ & $25 \%$ \\
\hline \multirow{2}{*}{$I_{\mathrm{w}}=I_{\max }$} & $I_{\min }$ & $25 \%$ \\
\hline & $I_{\max }$ & $75 \%$ \\
\hline$I_{\mathrm{w}}=I_{\min }=I_{\max }$ & $I_{\mathrm{w}}$ & $100 \%$ \\
\hline
\end{tabular}

If $I_{w}$ was not given, then each intensity between $I_{\min }$ and $I_{\max }$ was equally weighted. If $I_{\min }$ or $I_{\max }$ was not given, it was assumed to be $I_{w}-1$, and $I_{w}+1$, respectively. If $I_{w}$ was half a unit (in some cases with data from other agencies than SED), it was in a first step randomly changed into either the next upper or the next lower integer intensity; $I_{\min }$ and $I_{\max }$ were adapted, if necessary, in order to be $\leq I_{w}$, or $\geq I_{w}$, respectively. Based on this probability model, the intensity of each data point was randomly assigned. We then applied the BW method to all the resampled datasets to assess location and magnitude for the above-mentioned strategies (alpine strategy only in case of alpine event), resulting in a distribution of locations and magnitudes for the event that allowed the analysis of the parameter uncertainty. We computed distribution of locations and magnitudes for the events listed in Table 5, without taking into account our historical knowledge.

The uncertainty in location is assessed through the analysis of the distributions of epicenter locations defined by the position of the minimum RMS in the BW approach. The catalogue location, in most of the cases, corresponds to the center of the grid search area. A high percentage of the RMS locations of the resampled datasets are within a distance less than $20 \mathrm{~km}$ from the catalogue epicenter. Figure 12 provides two examples of the distributions of 
Fig. 12 Distribution of possible epicenter locations from the bootstrap technique for the strategies: dataset 1, allint, fixeddepth; dataset 1, top 3, fixeddepth; dataset 2 alpine, variable-depth, $h=10 \mathrm{~km}$ (this last only for event 1770.03.20). The black dot corresponds to the catalogue location. Contour lines represent the distance to the catalogue location (10 and $20 \mathrm{~km}$ ). The percentage of locations within the $20-\mathrm{km}$ distance from the catalogue location is: a $95 \%$, b $80 \%$
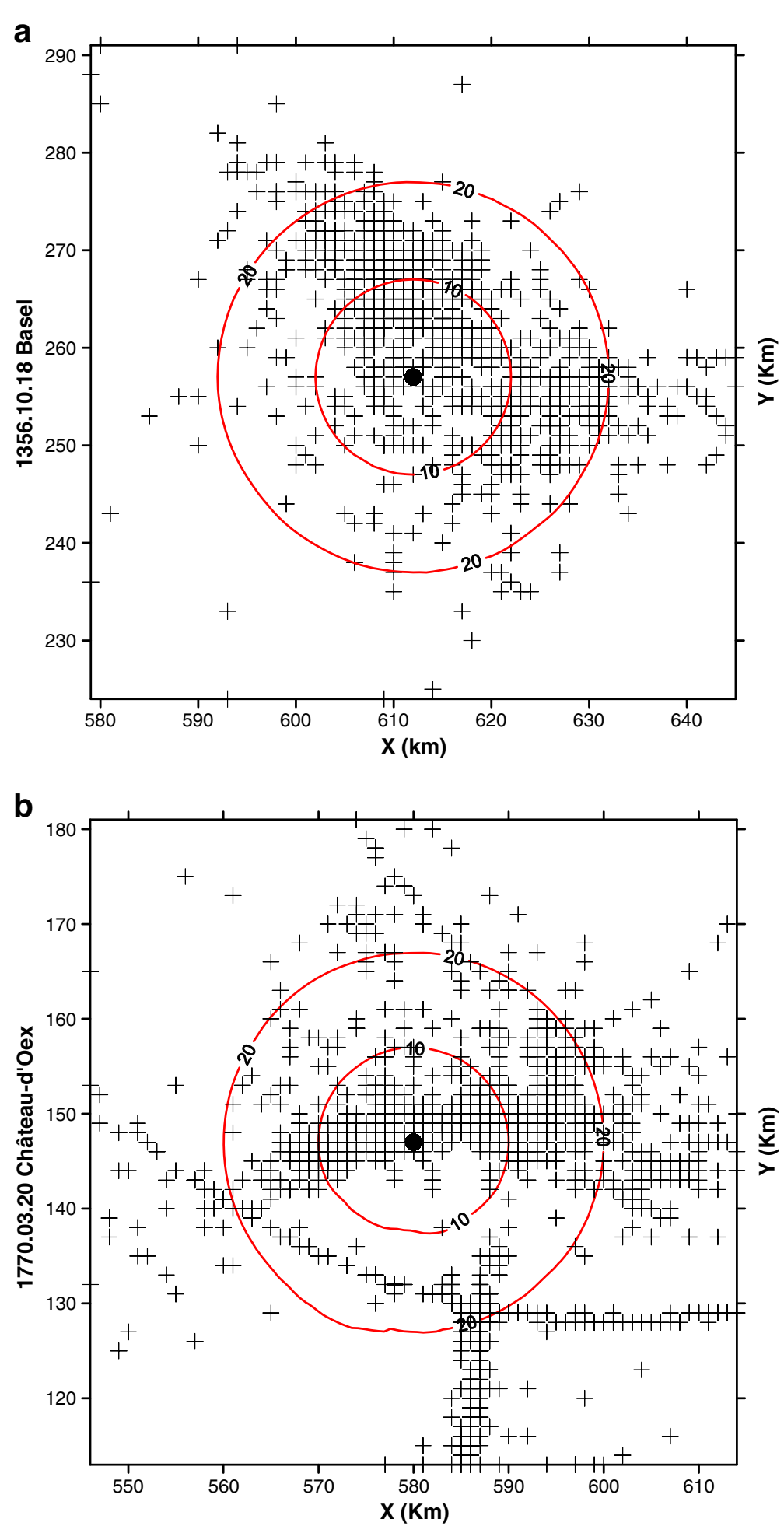

epicenter locations. These two cases represent a good macroseismic field (event 1356.10.18) and a poor one (event 1770.03.20); see Table 7. The uncertainty in location in the ECOS-09 catalogue is equivalent to $2 \sigma$. For most of the events that were assessed with the BW method, this uncertainty corresponds to either $20 \mathrm{~km}$ 
Table 7 Mean and standard deviation of the magnitude distributions (at catalogue location and minimum RMS locations) applied to resampled macroseismic fields using the bootstrap technique

\begin{tabular}{|c|c|c|c|c|c|c|c|c|c|c|c|}
\hline Year & Month & Day & Hour & $\begin{array}{l}\text { Event name } \\
\text { in ECOS-02 }\end{array}$ & $\begin{array}{l}\text { Number } \\
\text { IDPs }\end{array}$ & $M_{\mathrm{w}}(\text { bestmag })^{\mathrm{a}}$ & $\begin{array}{l}M_{\mathrm{w}} \\
\text { ECOS }-02^{\mathrm{b}}\end{array}$ & $\begin{array}{l}\text { Mean } M_{\mathrm{w}} \\
\text { bootstrap }\end{array}$ & $\begin{array}{l}\text { SD } \\
\text { bootstrap }\end{array}$ & $\begin{array}{l}M_{\mathrm{W}} \text { from } \\
\text { BW method }\end{array}$ & $\begin{array}{l}M_{\mathrm{w}} \\
\text { ECOS-09 }\end{array}$ \\
\hline 1295 & Sept & 03 & 00 & Churwalden & 9 & - & 6.5 & 6.4 & 0.33 & 6.2 & 6.2 \\
\hline 1356 & Oct & 18 & 21 & Basel & 47 & - & 6.9 & 6.6 & 0.12 & 6.6 & 6.6 \\
\hline 1584 & Mar & 11 & 11 & Aigle & 27 & - & 6.4 & 5.9 & 0.17 & 5.9 & 5.9 \\
\hline 1601 & Sept & 18 & 1 & Unterwalden & 67 & - & 6.2 & 5.9 & 0.15 & 5.9 & 5.9 \\
\hline 1685 & Mar & 8 & 19 & Mittelwallis & 9 & - & 6.1 & 5.6 & 0.17 & 5.3 & 5.3 \\
\hline 1755 & Dec & 9 & 13 & Brig-Naters & 128 & - & 6.1 & 5.7 & 0.19 & 5.7 & 5.7 \\
\hline 1770 & mar & 20 & 15 & Château-d'Oex & 8 & - & 5.7 & 5.2 & 0.21 & 5.2 & 5.2 \\
\hline 1855 & Jul & 25 & 11 & Törbel & 265 & - & 6.4 & 6.2 & 0.16 & 6.2 & 6.2 \\
\hline 1905 & Dec & 25 & 17 & Domat-Ems & 99 & 4.7 & 4.8 & 4.79 & 0.16 & 4.8 & 4.7 \\
\hline 1905 & Dec & 26 & 0 & Tamins & 96 & - & 5.1 & 4.77 & 0.17 & 4.7 & 4.7 \\
\hline 1913 & Jul & 20 & 12 & Ebingen & 880 & - & 5.2 & 5.0 & 0.13 & 5.0 & $5.2^{\mathrm{e}}$ \\
\hline 1929 & Mar & 1 & 10 & Bioley-Magnoux & 64 & 5.0 & 5.3 & 4.74 & 0.23 & 4.7 & 5.0 \\
\hline 1946 & Jan & 25 & 17 & Ayent & 602 & 5.8 & 6.1 & 5.73 & 0.12 & 5.7 & 5.8 \\
\hline 1946 & May & 30 & 3 & Ayent & 404 & 5.5 & 6.0 & 5.42 & 0.06 & 5.4 & 5.5 \\
\hline 1978 & Sept & 3 & 5 & Ebingen & 1,120 & 5.5 & 5.15 & 5.47 & 0.23 & 5.3 & 5.5 \\
\hline 1991 & Nov & 20 & 1 & Vaz/GR & 322 & 4.7 & 4.6 & 4.65 & 0.18 & 4.6 & 4.7 \\
\hline
\end{tabular}

The event names refer to the names in the 2002 catalogue. They changed in ECOS-09

${ }^{\mathrm{a}} M_{\mathrm{w}}$ (bestmag): $M_{\mathrm{w}}$ derived from instrumental recordings by Bernardi et al. (2005)

${ }^{\mathrm{b}} M_{\mathrm{w}}$ in the ECOS-02 catalogue

${ }^{\mathrm{c}} M_{\mathrm{w}}$ estimated with the BW method for ECOS-09

${ }^{\mathrm{d}} M_{\mathrm{w}}$ in the ECOS-09 catalogue

${ }^{\mathrm{e}}$ Magnitude from foreign catalogue

radius (we defined it as error class 3 in ECOS-09) or $50 \mathrm{~km}$ (defined as error class 4 in ECOS-09; equal to $2 \sigma$ ) around the epicenter location. The smaller error was assigned when the intensity field is considered to be sufficiently complete in terms of azimuth coverage, absence of gaps in historical information, and the number of IDPs with a large intensity range. For events that had an insufficient number of IDPs to apply the BW technique, the error class was chosen according to the available macroseismic and historical information.

We have computed the distribution of the magnitudes at the catalogue location $\left(M_{\text {cat }}\right)$ and at the minimum RMS location $\left(M_{\mathrm{RMS}}\right)$ for all strategies and for the 1,000 resampled datasets. Examples for two events are shown in Fig. 13. We then analyzed the distributions and estimated the mean and standard deviation for the selected events (see Table 7). The uncertainty of the magnitude is given in terms of $1 \sigma$. The standard deviations can be reduced if we only consider the distribution of magnitude at the cata- logue location, which is equivalent to the use of historical information. In Fig. 13, the distributions of magnitudes are shown for two different events. These distributions include the magnitude estimates obtained for all resampled sets and using strategies: dataset 1 allint, fixed-depth; dataset 1 top 3 fixeddepth, and dataset 2 alpine variable-depth $h=10 \mathrm{~km}$ (this last strategy only applied to event 1770.03.20). The contribution of varying the depth to the distribution is within the range of the overall distributions. We interpret the uncertainty of magnitude from bootstrap resampling as a lower bound of the uncertainty, as it does not account for the information missing in the macroseismic field, nor from uncertainty introduced by uncertainty in the parameters of the calibration datasets. Finally, we have tested that the magnitudes are normally distributed. We have addressed the uncertainty related to the calibration methods by testing the performance of the BW technique for the different ECOS-09 strategies. 
Fig. 13 Distributions of $M_{\text {cat }}$ and $M_{\text {RMS }}$ for the 1,000 bootstrap resampled datasets of a 1356.10.18 Basel event and b 1770.03.20 Chateau d'Oex event (see Table 7)
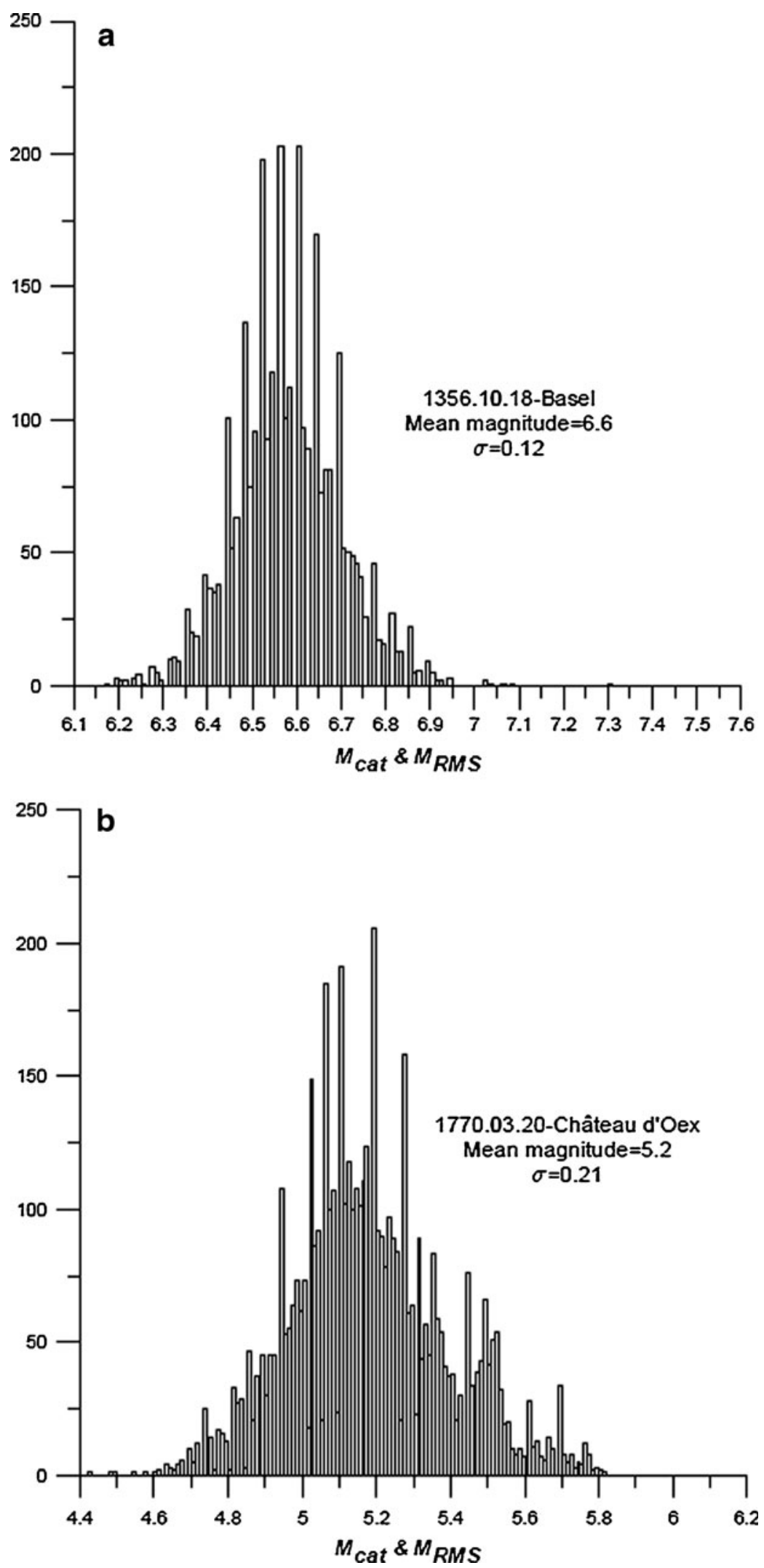


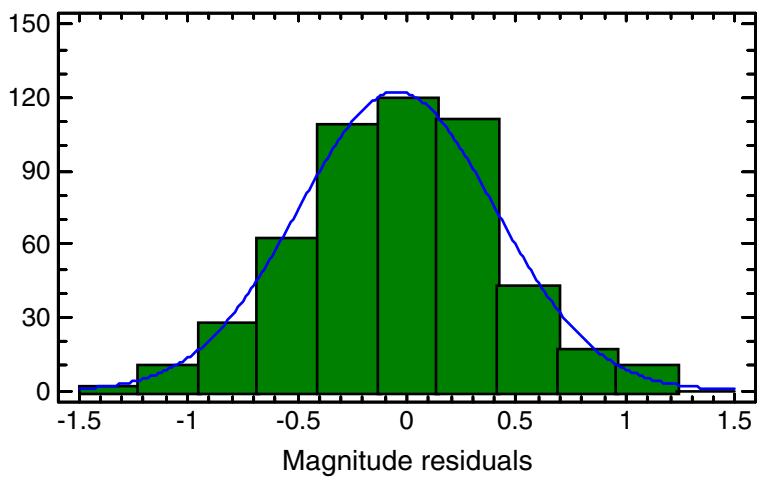

Fig. 14 Histogram and fitted normal probability density function of the magnitude residuals obtained applying the five ECOS-09 strategies at the catalogue and minimum RMS locations for all events of the dataset 1 with $M_{w}$ (bestmag). Depth is $h=10 \mathrm{~km}$ for the variable depth strategies

Figure 14 shows the magnitude residual distribution, difference between the magnitude assessed by BW and the $M_{w}$ (bestmag) of the calibration dataset. We have computed such magnitude residuals for all five ECOS-09 strategies (depth is fixed to $10 \mathrm{~km}$ for variable-depth strategies), for the three different weighting schemes tested during the macroseismic magnitude calibration, as well as for the different cutoff distances applied in BW. The residuals correspond to $M_{\text {cat }}$ and $M_{\mathrm{RMS}}$ for each event of calibration dataset 1 with known instrumental magnitude.

In Fig. 14, 1 standard deviation corresponds to 0.45 magnitude units. This standard deviation relates to the fact that it has taken into account all minimum RMS locations and therefore excludes any historical information. Events with $M_{w}$ (bestmag) smaller than 4.0 are also included, although they do not play an important role in the historical assessment; they significantly contribute to the tail of the distribution.

The residuals between BW magnitude assessed for all strategies and the $M_{w}$ (bestmag) of the calibration dataset are considered to be a measure of the epistemic uncertainty derived from modeling, if the $M_{w}$ (bestmag) would be without error. This error in the magnitudes $M_{w}$ (bestmag) makes the distribution broader. We therefore consider the distribution of these residuals to reflect an expected upper bound of overall uncertainty. In summary and taking into consideration the different estimations of uncertainty discussed here, an estimate of magnitude uncertainty in terms of $1 \sigma$ would be in the range 0.1 to 0.45 magnitude units.

In ECOS-09 catalogue, uncertainties are given as $2 \sigma$. For most of the events that were assessed with the BW method, the chosen magnitude uncertainty in the catalogue corresponds to 0.5 magnitude units (error class 2) or 1.0 magnitude unit (error class 3 ). For events with only few IDPs and for which an assessment with BW method was not possible, we assigned the error class 3 or larger, or class 0 (unknown).

\section{Conclusions}

The Swiss Seismological Service has addressed the reassessment of historical events within the catalogue compilation for Switzerland ECOS-09. To accomplish this reassessment, a modified Bakun and Wentworth method was selected as the main method. The main reason for the choice of the method is its flexibility and the possibility of including a priori historical information for the epicenter location. Historical earthquakes have been parameterized (epicentral location, magnitude, and depth when possible) following the similar two-step procedure as applied for the previous catalogue version ECOS-02. The derivation of the distance decay of macroseismic intensity is decoupled from the magnitude estimation (magnitude to standardized intensity calibration). We tested different models of intensity decay with distance in terms of formulation and variables involved. We have calibrated those models using three different calibration datasets (one of them includes regionalization). A calibration dataset with instrumentally derived moment magnitudes has been used for the calibration of a linear relation between an intercept intensity and magnitude. Analysis of the performance of calibration parameters and procedures resulted in the following rules: A distance weighting of the intensity data points was applied; intensities smaller than 3 were removed; the intensity representation used is "no binning". According to the variables involved in the attenuation model, three calibration strategies were analyzed: fixed-depth (set to $10 \mathrm{~km}$ ); variable-depth (depth to vary between 3 and $25 \mathrm{~km}$ ); mixed-strategy where the focal depth is fixed for those calibration events where it is known from instrumental data, and it is free for those with unknown depth. The magnitude calibration was also performed for three different intensity weighting schemes. The model with logarithmic, and linear distance decay was finally selected as the best performing ECOS-09 intensity attenuation model. Based on the perfor- 
mance of the calibration of ECOS-09 model over the different calibration datasets and depth strategies, we have defined five strategies that performed best and that have been applied for the assessment of earthquake parameters of historical events. Therefore, the assessment has been carried out applying four calibrated nonregional attenuation models and one regional Alpine model. Two models are for depth fixed at $10 \mathrm{~km}$, while three strategies are with variable depth.

Finally, the origin of uncertainties was assessed quantitatively by defining bounds of the uncertainties for magnitude and location. Bootstrapping has been used to assess a lower bound for the uncertainty. Uncertainties may arise from the distribution of the intensity data points such as, irregular azimuth coverage due to national borders or gaps in historical information, incompleteness, and uncertainty of the single intensity point. We have also assessed the uncertainty due to methodology (calibration models) in the test of the performance of the BW technique for the five ECOS-09 strategies. Finally, an uncertainty in location and magnitude is proposed for the historical earthquakes with sufficient number of intensity data points. Regarding the estimate of the magnitude uncertainty, most events are given 0.5 magnitude units or 1.0 magnitude unit error at 2 standard deviations. This may be compared with error estimates for $M_{\mathrm{w}}$ derived from magnitude conversions of instrumental catalogues of the twentieth century with errors in a similar range. The example shows that, based on the available historical macroseismic data, earthquake parameters can be assessed at precisions that are close to the ones derived from instrumental data in the early instrumental period. This highlights the importance of careful assessment of macroseismic information for Probabilistic Seismic Hazard Analysis (PSHA).

Acknowledgements This research was supported partly by Swissnuclear in project PRP (PEGASOS Refinement Project) and by the European project NERIES (Network of Research Infrastructures for European Seismology, task NA4-'Distributed Archive of Historical Earthquake Data').

\section{References}

Bakun WH, Scotti O (2006) Regional intensity attenuation models for France and the estimation of magnitude and location of historical earthquakes. Geophys J Int 164 (3):596-610
Bakun WH, Wentworth CM (1997) Estimating earthquake location and magnitude from seismic intensity data. Bull Seismol Soc Am 87(6):1502-1521

Bakun WH, Johnston AC, Hopper MG (2003) Estimating locations and magnitudes of earthquakes in Eastern North America from modified Mercalli intensities. Bull Seismol Soc Am 93(1):190-202

Bernardi F, Braunmiller J, Giardini D (2005) Seismic moment from regional surface-wave amplitudes: applications to digital and analog seismograms. Bull Seismol Soc Am 95 (2):408-418. doi:10.1785/0120040048

Blake A (1941) On the estimation of focal depth from macroseismic data. Bull Seismol Soc Am 31(3):225231

Braunmiller J, Deichmann N, Giardini D, Wiemer S, SED Magnitude Working Group (2005) Homogeneous moment-magnitude calibration in Switzerland. Bull Seismol Soc Am 95(1):58-74. doi:10.1785/0120030245

Fäh D, Giardini D, Bay F, Bernardi F, Braunmiller J, Deichmann N, Furrer M, Gantner L, Gisler M, Isenegger D, Jimenez MJ, Kaestli P, Koglin R, Masciadri V, Rutz M, Scheidegger C, Schibler R, Schorlemmer D, SchwarzZanetti G, Steimen S, Sellami S, Wiemer S, Wossner J (2003) Earthquake catalogue of Switzerland (ECOS) and the related macroseismic database. Eclogae Geologicae Helvetiae 96:219-236

Fäh D, Giardini D, Kästli P, Deichmann N, Gisler M, Schwarz-Zanetti G, Alvarez-Rubio S, Sellami S, Edwards B, Allmann B, Bethmann F, Wössner J, Gassner-Stamm G, Fritsche S, Eberhard D (2011) ECOS-09 Earthquake Catalogue of Switzerland Release 2011. Report and Database. Public catalogue, 17.4.2011. Swiss Seismological Service ETH Zürich, Report SED/ RISK/R/001/20110417

Gasperini P (2004) BOXER user guide, version 3.3

Gasperini P, Bernardini F, Valensise G, Boschi E (1999) Defining seismogenic sources from historical earthquake felt reports. Bull Seismol Soc Am 89(1):94-110

Gisler M, Fäh D (2011) Grundlagen des Makroseismischen Erdbebenkatalogs der Schweiz (MECOS) 1681-1878. Herausgegeben vom Schweizerischen Erdbebendienst. vdf, Zürich

Gómez Capera AA (2006) Seismic hazard map for the Italian territory using macroseismic data. Earth Sci Res J 10 (2):67-90

Gómez Capera AA, Meletti C, Musson R, Stucchi M (2009) Deliverable D5-the European Earthquake Catalogue (demo version) 1000-1600. Part 1-the NA4 calibration initiative. NA4 distributed archive of historical earthquake data. Sixth Framework Programme. EC project number:026130

Hinzen K-G, Oemisch M (2001) Location and magnitude from seismic intensity data of recent and historic earthquakes in the Northern Rhine Area, Central Europe. Bull Seismol Soc Am 91(1):40-56

Kövesligethy R (1906) Mathematikai és Természettudomanyi Értesitö 24:349-368 (in Hungarian)

Musson R, Jiménez M, Gómez Capera AA (2008) Macroseismic estimation of earthquake parameters. Seismology and Geomagnetism Programe-open report OR/08/004. British Geological Survey, 55pp 
Pasolini C, Gasperini P, Albarello D, Lolli B, D'Amico V (2008) The attenuation of seismic intensity in Italy, part I: theoretical and empirical backgrounds. Bull Seismol Soc Am 98(2):682-691

Schwarz-Zanetti G, Fäh D (2011) Grundlagen des Makroseismischen Erdbebenkatalogs der Schweiz (MECOS) 1000-1680. Herausgegeben vom Schweizerischen Erdbebendienst. vdf, Zürich

Schweizerische Erdbebenkommission (1879-1912) Jahresberichte Schweizerischer Erdbebendienst (1913-1963) Jahresberichte

Schweizerischer Erdbebendienst: Jahresberichte des Schweizer Erdbebendienstes
Stromeyer D, Grunthal G (2009) Attenuation relationship of macroseismic intensities in Central Europe. Bull Seismol Soc Am 99(2A):554-565. doi:10.1785/0120080011

Stucchi M (2010) Deliverable D10-NA4 European Earthquake Catalogue 1000-1963, M>5.8. NA4 distributed archive of historical earthquake data. Sixth Framework Programme. EC project number:026130

Swiss Seismological Service (2002) ECOS Earthquake Catalogue of Switzerland. Swiss Seismological Service ETH Zürich. http://www.seismo.ethz.ch Accessed on: July 2008)

Untersuchungen zu Erdbeben (1972-1974) Bulletins, Berichte, Zürich 